\title{
A General Phenomenon of Spontaneous Amplification of Optical Purity under Achiral Chromatographic Conditions
}

\author{
K. Michał Pietrusiewicz ${ }^{1}\left(\mathbb{D}\right.$, Mariusz Borkowski $^{1,2}{ }^{\mathbb{D}}$, Dorota Strzelecka ${ }^{1}$, Katarzyna Kielar $^{1}$, \\ Wioleta Kicińska ${ }^{1}$, Sergei Karevych ${ }^{1}$, Radomir Jasiński ${ }^{3}$ and Oleg M. Demchuk ${ }^{1,4, * \mathbb{D}}$ \\ 1 Faculty of Chemistry, Maria Curie-Skłodowska University, 2 M. Sklodowskiej-Curie Square, \\ 20-031 Lublin, Poland; Kazimierz.Pietrusiewicz@poczta.umcs.lublin.pl (K.M.P.); \\ ncborkow@cyf-kr.edu.pl (M.B.); dorin538@gmail.com (D.S.); kasiak1005@teln.pl (K.K.); \\ kicinskawioleta@gmail.com (W.K.); kackac16@gmail.com (S.K.) \\ 2 Institute of Catalysis and Surface Chemistry, Polish Academy of Sciences, 8 Niezapominajek Str., \\ 31-155 Cracow, Poland \\ 3 Institute of Organic Chemistry and Technology, Cracow University of Technology, 24 Warszawska Str., \\ 31-155 Cracow, Poland; radomir@indy.chemia.pk.edu.pl \\ 4 Pharmaceutical Research Institute, 8 Rydygiera Str., 01-793 Warsaw, Poland \\ * Correspondence: O.Demchuk@IFarm.eu
}

Received: 2 April 2019; Accepted: 15 May 2019; Published: 17 May 2019

\begin{abstract}
This work explores the behavior of chiral compound mixtures enriched in one of the enantiomers whilst a typical chromatography on the achiral stationary phase is employed. The influence of several factors, such as the eluent composition, ratio of the compound to the stationary phase, and the initial enatiomeric purity of the compound used on the distribution of the enantiomers in the collected chromatographic fraction, was studied. The obtained results indicate that the phenomenon of Self Disproportionation of Enantiomer (SDE) occurred in all cases, and some of the collected fractions got higher optical purities than the initial one. Thus, achiral column chromatography could be applied in some cases as the simplest approach for chiral purification. Based on the experimental results and DFT calculations, an alternative concept explaining the SDE phenomenon was proposed. Due to its generality and simplicity, SDE may also be responsible for the formation of the first chiral non-racemic compounds on the early Earth.
\end{abstract}

Keywords: enantiomer self-disproportionation; SDE; achiral stationary phase; homochiral and heterochiral aggregates; chiral separation; chirality; genesis of life chirality

\section{Introduction}

The mystery of life on Earth is closely connected with the phenomenon of the chiral nature of all living organisms (human beings, animals, plants, insects, etc.). Chirality, as a fundamental property of 3-dimensional objects (including many molecules and ions) to be non-superinposable on their mirror images, is imprinted in such basic building blocks of life as amino acids and sugars; it is further reflected by the chirality of more complex biochemical objects such as DNA and eventually by the chirality of entire bodies [1,2]. Interestingly, amino acids [3] and sugars [4] derived from natural sources are non-racemic and are constituted of single stereoisomers. At the same time, according to the fundamental chemical principles the formation of non-racemic chemical compounds is impossible when the chiral component of the chemical reaction (substrate, catalyst, medium) is absent [5]. Despite its crucial value for the understanding of the origin of life, the origins of the chirality of molecules of life are still unknown [6]. Apart from the creationist approach stating that "the only possible way 
for unique homochirality to exist in the chiral biochemical molecules found in living organisms is for those biomolecules to have been created with unique homochirality when that organism was first created. Just as a fingerprint identifies its creator" [7], there are several other theories trying to address this issue [8]: partial photochemical decomposition of the racemic mixtures of chiral compounds in the interstellar clouds caused by polarized cosmic irradiation $[9,10]$; photochemical reactions in the primordial soup influenced by the Sun's light polarized by passing though the Earth atmosphere [11,12]; the effect of the parity-symmetry violation of the weak force [13]; the stereochemical outcome of the chemical reactions influenced by a magnetic field [14]; asymmetric catalysis mediated with chiral minerals e.g., single quartz crystals $[15,16]$; stereoselective crystallization of a single enantiomer from the racemic mixture $[17,18]$. Since the formation of both enantiomers is statistically equal, all the variety of the chiral compounds on Earth should have originated from almost a single act of creation. Moreover, in all those rare cases, mixtures with only an extremely low excess of one of the enantiomers could be formed. Thus, the major question of the chirality genesis is how-from an almost racemic mixture of chiral probiotic compounds formed accidentally, once or few times only-an optically pure substance could have been spontaneously created. The process of the spontaneous enrichment of the enantiomer mixture and the generality of this phenomenon could be studied on the example of several typical benchmark asymmetric syntheses where some enantio-enriched mixture of the chiral compounds is formed.

As a result of the significant progress made in the last few decades, the asymmetric catalysis has become the basic tool of synthetic organic chemistry used to obtain modern medicines [19], and cosmetics [20], plant protecting substances [21], and variety of functional materials [22,23]. Valuable features of those kinds of transformations include: the highest atom economy (thus, only one of two possible enantiomers are usually desirable); a short synthetic pathway because of the elimination of chiral derivatisation and racemic mixture separation steps; mild reaction conditions; wide substitution pattern toleration; and many others-all of the above made these transformations highly attractive economically. Therefore, they draw the attention of both academics and the industry. The importance of this field has also entailed the development of methods for the determination of the enantiomeric composition of the obtained products directly or after derivatisation by chiral reagents. There are the classical measurement of optical rotation power [24,25], chiral chromatographic approaches [26,27], NMR methodologies utilising chiral shifting reagents [28-31] and chiral derivatisation reagents [32-34].

In the majority of cases, the determination of the enantiomeric composition of the mixtures of isomeric products obtained in the reaction is performed after preliminary purification of the sample studied. Distillation is only marginally useful in the case of polysubstituted organic materials. Such routine purification techniques as crystallisation and sublimation cannot ensure the invariability of the enantiomeric composition in a rough reaction mixture and isolated substance because of a widely observed enantiomer self-disproportionation phenomenon (SDE). SDE was defined in 2006 as a transformation of an enantiomerically enriched system resulting in the formation of fractions with different, in comparison to the initial enantiomeric distribution [35]. The SDE phenomenon is attributed to two distinct models of the intermolecular interaction between the enantiomers of a chiral compound. These are homochiral $(R, R$ or $S, S)$ and heterochiral $(R, S)$-associations (Figure 1$)$ which are present in a not completely racemic mixture and are largely responsible for the nonlinear behavior of the optical rotation $[35,36]$. 


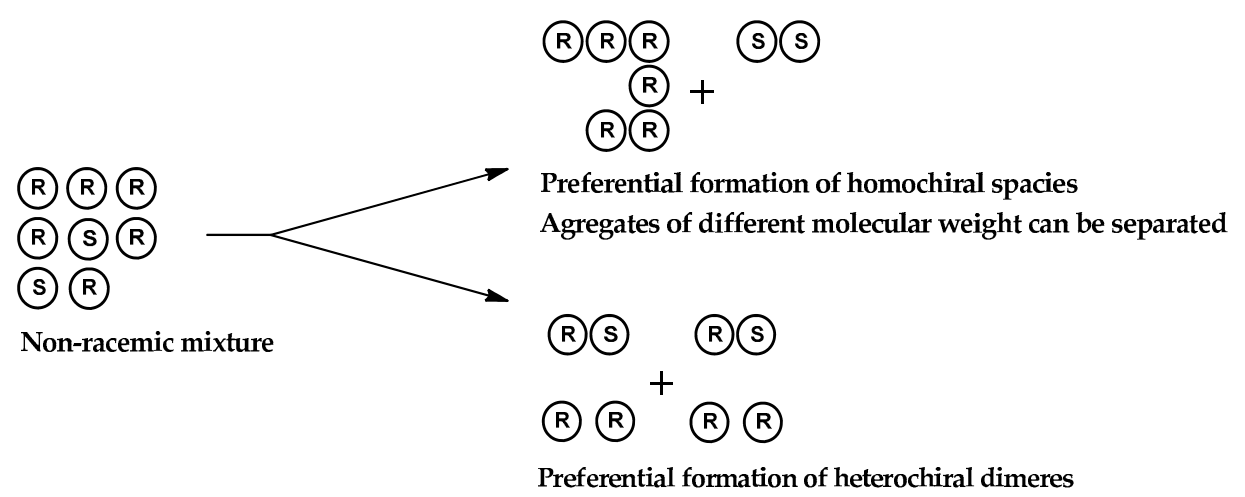

Figure 1. Preferential formation of homochiral or heterochiral aggregates in the solution of optically enriched compounds.

Thus, column chromatography is usually the method of choice for the purification of reaction mixtures in laboratory practice and it is extensively used for the preparation of analytical samples from different asymmetric reactions. For a long time, the chemical community accepted the assumption that, with very few exceptions [37], the ratio of enantiomers would not be significantly changed during chromatographic purification, unless some other chiral players such as chiral solvents or additives or a chiral stationary phase are used. Recent studies indicate that this rule could be broken in the case of chiral fluorinated compounds because of notable chiral self-aggregation promoted by a strong dispersive interaction of fluorous tails $[35,38]$. Rare literature examples also suggest that a partial separation of the racemate from the enantiomer could be observed for some other enantiomericaly enriched compounds subjected to achiral chromatographic purification via regular gravity driven chromatography on silica gel [39-42], or HPLC [43], MPLC [37], flash [44], or other chromatographic purification techniques [45]. However, this possibility is still usually neglected in routine chromatographic purification on regular achiral stationary phases.

Herein, we would like to demonstrate that SDE is a common phenomenon observed during the achiral low-pressure column chromatography of the chiral compounds obtained in benchmark asymmetric reactions such as allylic substitution, [46,47] cross-coupling [48-52] and organocatalytic aldol condensation $[53,54]$ as well as other important asymmetric transformations. Thus, such processes may play a significant role in the course of the chiral, non-racemic, pro-biotic compound formation.

\section{Materials and Methods}

All the chemicals used in this research were purchased from Sigma-Aldrich, Avantor Performance Materials Poland S.A. (formerly POCH S.A) and Merck. Analytical thin-layer chromatography (TLC) was performed using silica gel $60 \mathrm{~F}_{254}$ precoated plates $(0.25 \mathrm{~mm}$ thickness). Visualization of TLC plates was performed by means of UV light. NMR spectra were recorded on Bruker Avance 500 $\mathrm{MHz}$ spectrometers, and chemical shifts are reported in ppm, and calibrated to residual solvent peaks at $7.27 \mathrm{ppm}$ and $77.00 \mathrm{ppm}$ for ${ }^{1} \mathrm{H}$ and ${ }^{13} \mathrm{C}$ in $\mathrm{CDCl}_{3}$ or TMS as internal reference compounds. The enantiomeric compositions of the obtained fractions were determined by a chromatographic method (Shimadzu LCMS IT-TOF spectrometer) using chiral chromatographic columns Chiralpak ${ }^{\circledR}$ and Chiralcel ${ }^{\circledR}$ with the following dimensions: $150 \times 2.1 \mathrm{~mm}$, or by ${ }^{1} \mathrm{H} N \mathrm{NR}$ and ${ }^{13} \mathrm{C}$ NMR techniques using an appropriate chiral differentiating factor and solvents. The purification process and the study of the SDE phenomenon were conducted on a flash low pressure BUCHI chromatograph using $12 \times 150 \mathrm{~mm}$ PE columns packed by authors with $10 \mathrm{~g}$ spherical silica gel 60 with the particle size of 230-400 mesh as the stationary phase. The studied compounds were dissolved in $5 \mathrm{~mL}$ of an appropriate solvent and mixed with $1 \mathrm{~g}$ of silica gel. Next, the solvent was evaporated and the sample supported on silica was placed at the top of the column prepacked with silica. After that, the column was connected to the MPLC to be subjected to SDE studies. In the case of compounds $\mathbf{1}$ and $\mathbf{6}$, instead of supporting 
them on silica, the solutions of the studied compounds in $0.6 \mathrm{~mL}$ of mobile phase were injected onto the column prepacked with $11 \mathrm{~g}$ of silica and prewashed with the mobile phase. The fractions were automatically collected by $18 \mathrm{~mL}$ volume. The elution progress was monitored by TLC. All DFT calculations were performed using the "Prometheus" cluster in the "Cyfronet" computational center in Cracow. A new generation M062x [55]; [56] functional, implemented in the Gaussian 09 package [57], was used. This functional has been recently used by us to solve several similar problems [58-60]. All stationary structures have been optimized using the advanced $6-311++G(d, p)$ basis set and were characterized by only positive eigenvalues in their diagonalized Hessian matrices. For the optimized structures, thermochemical data for the temperature $\mathrm{T}=298 \mathrm{~K}$ and pressure $\mathrm{p}=1 \mathrm{~atm}$ were computed using vibrational analysis data. For the simulation of the solvent presence, the PCM model has been applied, similar to in previous our works $[59,60]$.

\section{Results and Discussion.}

In order to investigate the impact of a routine chromatographic purification process on the distribution of enantiomers in collected fractions, non-racemic mixtures of the studied compounds were obtained in the usual benchmark reactions.

\subsection{Study on the SDE of Dicarboxylic Acid Esters: (R)-Dimethyl [(2E)-1,3-Diphenyl-2-Propen-1-yl] Malonate (1)}

The asymmetric allylic substitution reaction leading to $(R)$-dimethyl [(2E)-1,3-diphenyl-2propen-1-yl] malonate (1) is a standard process used to assess the efficiency and compare the chiral transition metal complex based catalysts [59]. The product of this benchmark reaction was evaluated first. The reaction was carried out using (R)-(-)-BisNap-Phos [48] as a ligand and furnished crude product 1 (Scheme 1 ), which was subsequently subjected to the enantiomeric purity determination by an HPLC technique. The enantiomeric composition and $(R)$-absolute configuration of the product were determined by the peak integration and elution order from chiral HPLC using a Chiralcel ${ }^{\circledR}$ OD-H column [61]. The enantiomeric excess of the crude product was $49.0 \%$.

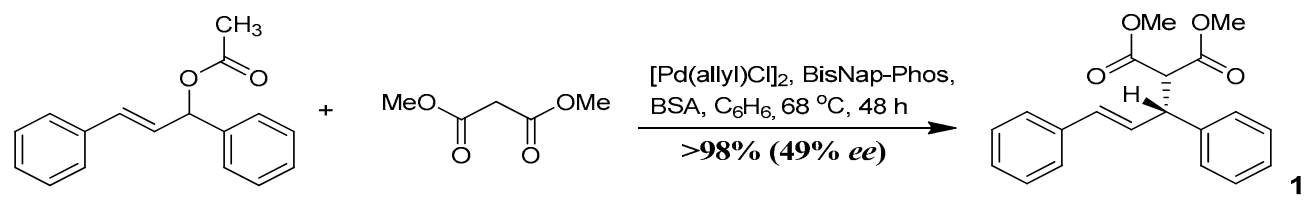

Scheme 1. Synthesis of (R)-dimethyl [(2E)-1,3-diphenyl-2-propen-1-yl] malonate (1).

Next, the product was chromatographically purified on the achiral stationary phase. The eluent system selection was based on the $R_{\mathrm{f}}$ values $(\sim 0.2)$ and hence the mixtures of hexane/acetone in the ratios of (99/1) and (99.7/0.3) were selected as mobile phases. $50.0 \mathrm{mg}$ of compound $\mathbf{1}$ was subjected to the purification process on an achiral $12 \times 150 \mathrm{~mm}$ column filled with $11.0 \mathrm{~g}$ silica gel. The separations were run at the eluent flow rate of $18 \mathrm{~mL} / \mathrm{min}$ and the collected fractions had the volume of $18 \mathrm{~mL}$. The fractions were collected from the moment when the eluted compound was detected by TLC. The enantiomeric compositions of consecutive fractions were determined by means of chiral HPLC with the application of a Chiralpak ${ }^{\circledR}$ AS-RH column and the mobile phase $\mathrm{H}_{2} \mathrm{O} / \mathrm{CH}_{3} \mathrm{CN}=55 / 45$, and $0.4 \mathrm{~mL} / \mathrm{min}$ flow (Figure S1). The Chiralpak ${ }^{\circledR} \mathrm{OJ}-\mathrm{RH}$ and Chiralcel ${ }^{\circledR} \mathrm{OD}-\mathrm{H}$ columns could also be used for this purpose. The elution profiles for both eluent systems are depicted in Figure 2 and Table S1. 


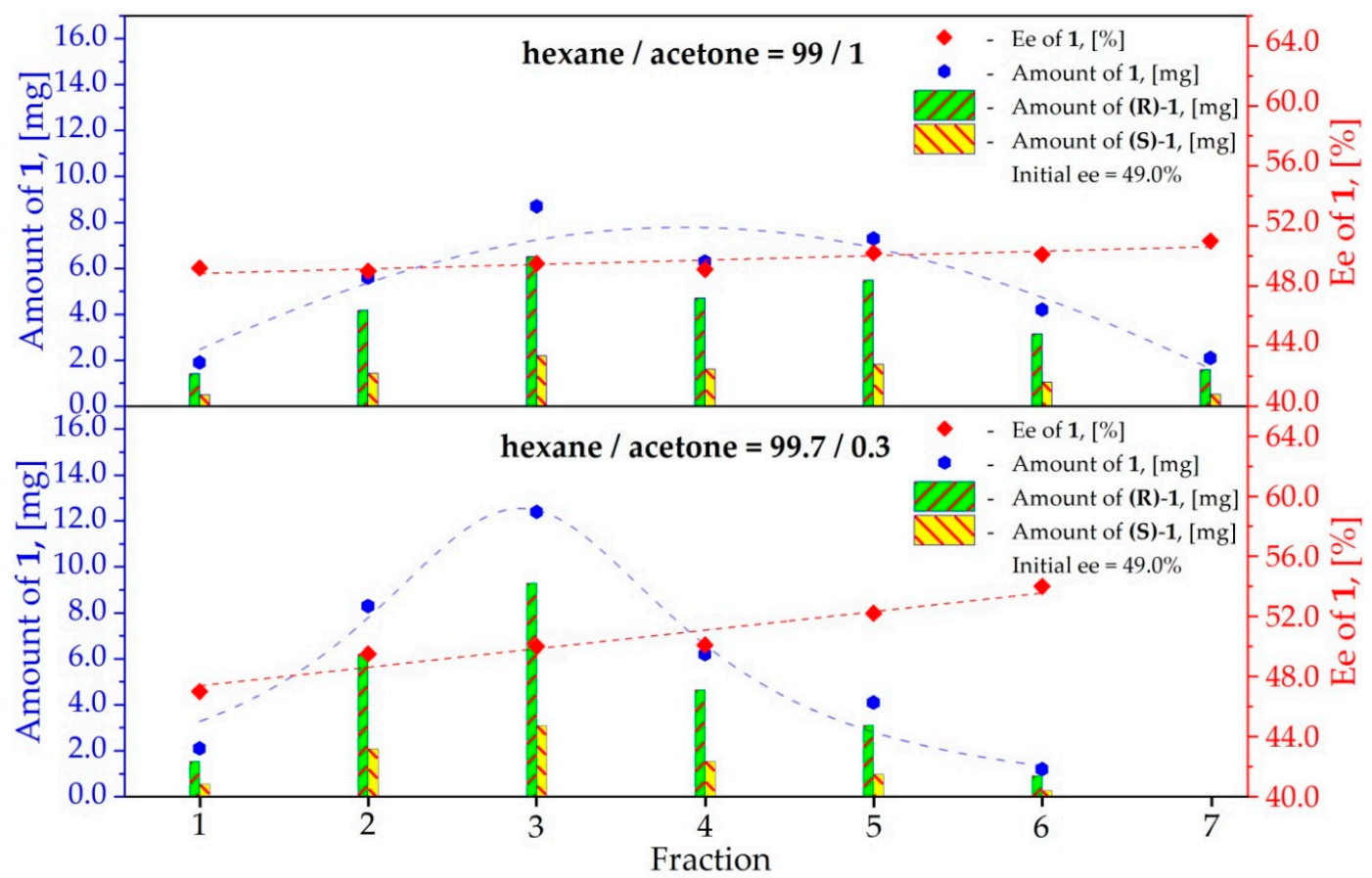

Figure 2. SDE of compound 1 in achiral MPLC conditions: the elution profiles. The fitting equation for hexane/acetone $=99 / 1$ is $y=0.018 x+49.429\left(R^{2}=0.851\right)$ and for hexane/acetone $=99.7 / 0.3$ is $y=2.099 x$ $+40.4\left(R^{2}=0.768\right)$.

The mobile phases were selected by the optimization process using the TLC method. Several possible solvent mixtures and pure solvents were tested in this process. In the next step, those mobile phases, for which the separation on the plates gave promising results were selected; these mobile phases were then tested in the column separation process. In those experiments, several mobile phases were examined and finally those for which the separation result was good enough were obtained and results obtained in the context of the SDE phenomenon. This procedure of mobile phase optimization was used in a course of all further separations. In this case, in the optimization process, hexane/acetone with ratios of 99:1 and 99.7:0.3 was selected as mobile phase. It can be seen that in the course of the purification, the $e e$ values of the collected fractions increased slightly from the initial level of $49.0 \%$ for hexane/acetone $=99: 1$ and more significantly for hexane/acetone in the ratio of 99.7: 0.3 (to $51.0 \%$ and $54.0 \%$, respectively). The values of the SDE magnitude $(\Delta e e)$ parameter (defined as the difference between the highest and the lowest value of the enantiomeric excess of the collected fractions) equaled $1.8 \%$ and $7.0 \%$ for hexane/acetone $=99: 1$ and hexane/acetone $=99.7: 0.3$, respectively. These results indicate that the eluent used in the first series of the experiment was characterized by a polarity too high to allow effectively the occurrence of SDE. In contrast to the first series, it is noticeable that the utilization of a less polar eluent leads to obtaining a compound with a slightly higher $e e$ value in the latter fractions. These results immediately allowed us to state that the purification process of the crude reaction mixture on achiral silica gel may affect the ee value. Secondly, the SDE of compound 1 depends on the eluent composition used in the purification process; the less polar eluent facilitates the SDE phenomenon.

\subsection{Study on the SDE of Atropisomeric Biaryl: 2,2'-Dimethoxy-1,1'-Binaphthyl (2)}

Inspired by the initial results, we decided to investigate whether the SDE phenomenon may be applicable to the enantiopurification of 2,2'-dimethoxy-1,1'-binaphthyl (2) enriched in the (R)-isomer (Scheme 2). Compound 2 was obtained in a benchmark Suzuki-Miyaura reaction which is usually used to assess the efficiency of a palladium catalyst in cross-coupling reactions. The reaction that was run in the presence of $0.3 \%$ SDS and $\mathrm{Na}_{2} \mathrm{CO}_{3}$ in an aqueous medium at ambient temperature utilized 
the (S)-BisNap-Phos ligand $(1 \mathrm{~mol} \%)$ and $\mathrm{Pd}\left(\mathrm{C}_{6} \mathrm{H}_{5} \mathrm{CN}\right)_{2} \mathrm{Cl}_{2}$ pre-catalyst $(0.5 \mathrm{~mol} \%)$. The enantiomeric excess of 2 was determined by an HPLC technique using a Chiralpak ${ }^{\circledR}$ AS-RH column, and the mobile phase $\mathrm{H}_{2} \mathrm{O} / \mathrm{CH}_{3} \mathrm{CN}=50 / 50$ (Figure S2). The initial enantiomeric excess was assigned as $41.0 \%$ ee.

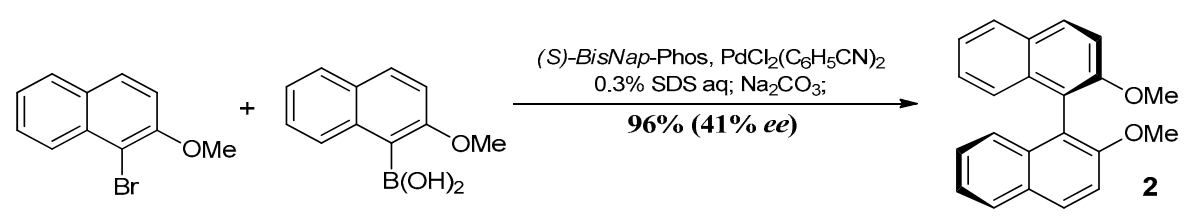

Scheme 2. Synthesis of (R)-2,2'-dimethoxy-1,1'-binaphthalene (2).

Further, $100.0 \mathrm{mg}$ of compound 2 was fractionated on a $12 \times 150 \mathrm{~mm}$ column filled with $10.0 \mathrm{~g}$ silica gel, using a mixture of hexane/ethyl acetate $=97: 3$ as an eluent. Considering the low solubility of compound 2 in the eluent, the compound was applied on the gel and loaded onto the column in this form. The separations were run at the eluent flow rate of $18 \mathrm{~mL} / \mathrm{min}$ and the collected fractions had the volume of $18 \mathrm{~mL}$. The SDE profile of compound 2 is shown in Figure 3 (the numeric data are given in Table S2). As one can see, the enantiomeric excess of the examined compound increased with the elution volume from $21.0 \% e e$ in fraction 32 (in which compound 3 was first detected by TLC) to $63.0 \% e e$ in the last fraction (fraction 52). The SDE magnitude ( $\triangle e e$ ) in the studied cases equaled $42.0 \%$. The chiral HPLC chromatograms of the first and last fractions are depicted in Figure S2. The final fraction (fraction 52) is $22.0 \%$ more enantiomerically enriched in comparison to the initial sample of $41.0 \%$ ee. Such highly significant value of $\Delta e e$ could be caused by the $\pi-\pi$ stacking interaction between the naphthyl moieties [62] as it has been previously observed for other types of substituted naphthalenes [63]. On the other hand, 2,2'-dimethoxy-1,1'-binaphthyl could easily undergo crystallization, and it is also theoretically possible that a multiple micro recrystallization process may have taken place on the column and eventually influenced the enantiomeric distribution in the collected fractions. In any case, the obtained results indicate a possibility of the enantiomeric enrichment of potentially crystalline compounds (or even a separation of the enantiomeric fraction from the racemate) using the methodology based on the achiral chromatographic technique.

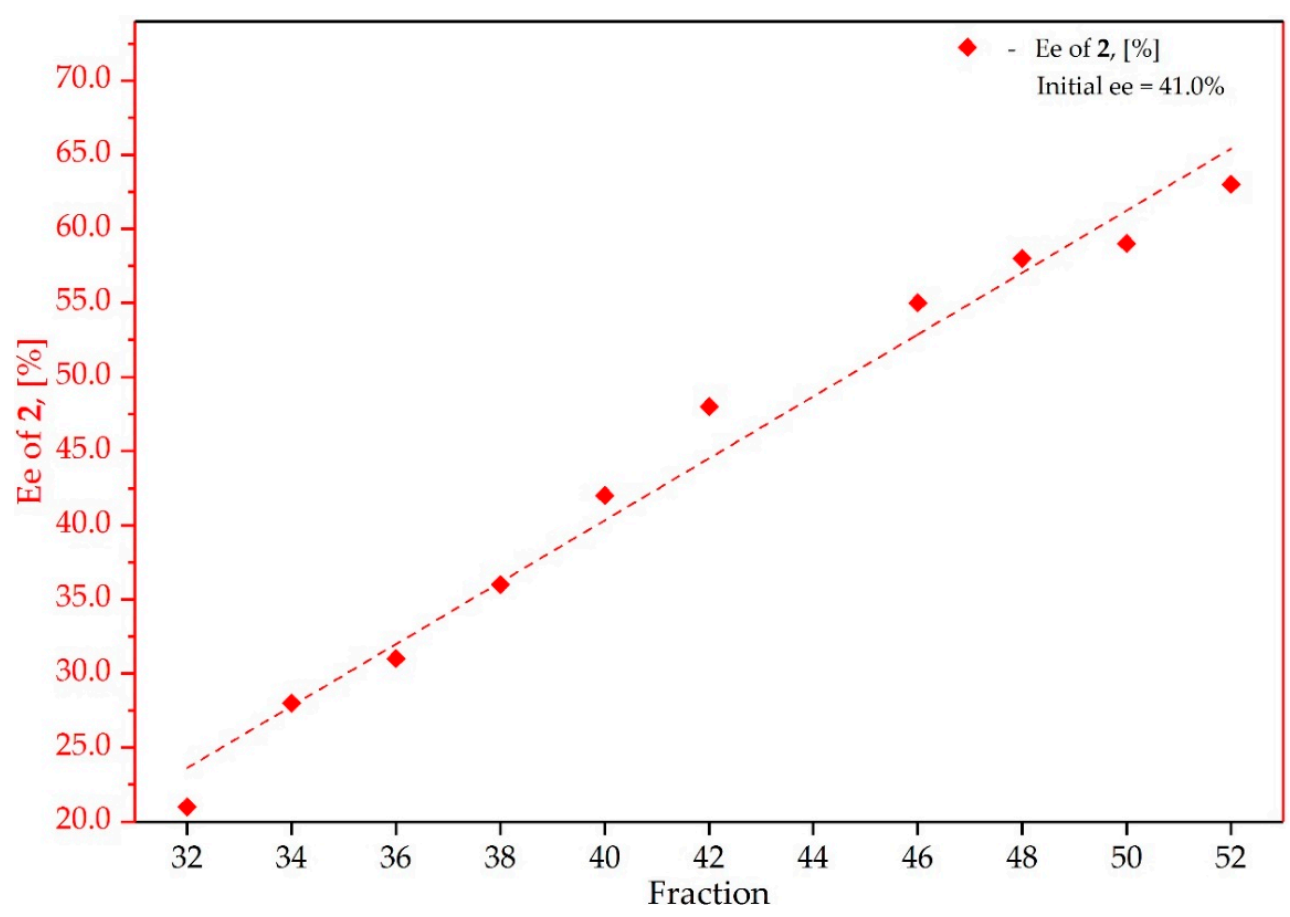

Figure 3. SDE of compound 2 in achiral MPLC conditions: the elution profile. The fitting equation is $y=2.089 x-46.242\left(R^{2}=0.979\right)$. 


\subsection{Study on the SDE of $\beta$-Hydroxy Ketone: (4R)-(4-nitrophenyl)-4-Hydroxy-2-Butanone (3)}

Taking into account a possible strong effect of the $\pi-\pi$ forces in the crystal net, we turned our attention to another class of organic compounds. The next chiral model compound (4R)-(4-nitrophenyl)-4-hydroxy-2-butanone (3) was synthesized under aldol condensation conditions (Scheme 3) [64] in a reaction between 4-nitrobenzaldehyde and technical grade acetone in the presence of $20 \mathrm{~mol} \%$ of $L$-proline. In this benchmark organocatalytic reaction the chiral organic molecule plays the role of a catalyst, which is more inherent to biomimetic processes. As a result, the reaction products are not contaminated with toxic metal impurities, the catalyst does not undergo decomposition, and in general, the process itself is compatible with the requirements of green chemistry, as well as chemical processes occurring on the early Earth. The optical purity of synthesized compound 3 was determined by an HPLC technique using a Chiralpak ${ }^{\circledR}$ AD-RH column, and the $\mathrm{CH}_{3} \mathrm{CN} / \mathrm{H}_{2} \mathrm{O}=30 / 70$ mobile phase [65] (Figure S3).
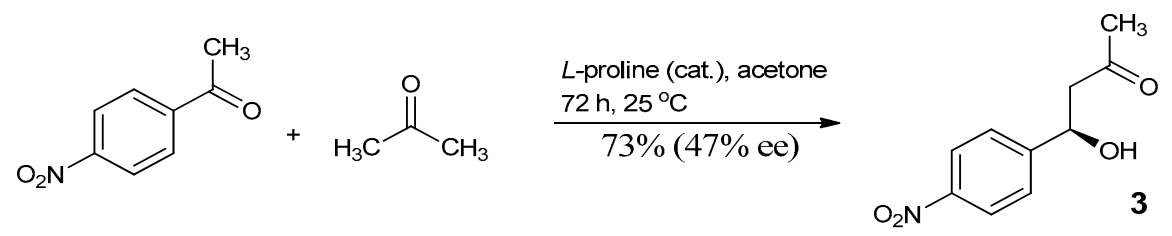

Scheme 3. Synthesis of (R)-4-hydroxy-4(nitrophenyl)butan-2-one (3).

The ratio of the amount of the studied compound to the amount of the stationary phase used may affect the efficiency of SDE. To explore this dependence three different quantities of $3(50.0 \mathrm{mg}$, $100.0 \mathrm{mg}$ and $150.0 \mathrm{mg}$ supported on $1.0 \mathrm{~g}$ of silica) were eluted with hexane/MTBE $=85: 15 \mathrm{in}$ flash chromatography conditions on a $12 \times 150 \mathrm{~mm}$ column filled with $10.0 \mathrm{~g}$ silica gel. On the basis of the obtained results (Table S3) the enantiomeric distribution profiles are presented in Figure 4. In all cases a clear decreasing dependence of the enantiomeric excess was observed. First fractions exhibited a higher enantiomeric enrichment in the $(R)$-isomer than the latter ones. The values of $\Delta e e$ were $23.6 \%, 17.2 \%$ and $16.8 \%$ for $50.0 \mathrm{mg}, 100.0 \mathrm{mg}$ and $150.0 \mathrm{mg}$ of the examined compound sample size, respectively. In the case of the sample with the smallest quantity of the compound the highest $e e$ value of $55.6 \%$ occurred in fraction 37 (first fraction in which compound 3 was detected by TLC), while the lowest in fraction 70 and equaled $32.0 \%$ ee. In comparison with the initial value of $47.0 \%$ ee, the first fractions were enantiomerically enriched in $(R)$-isomer by $8.6 \%$ (for a $50.0 \mathrm{mg}$ sample), $5.5 \%$ (for a $100.0 \mathrm{mg}$ sample), $1.8 \%$ (for a $150.0 \mathrm{mg}$ sample), while the last fractions were enantiomerically depleted by $15 \%$ (for the $50.0 \mathrm{mg}$ and $150.0 \mathrm{mg}$ sample), $10.6 \%$ (for a $50.0 \mathrm{mg}$ sample). As it can be seen in Figure 4, for $100.0 \mathrm{mg}$ and $150.0 \mathrm{mg}$ of the compound in the sample the SDE phenomenon occurred, yet to a lesser degree. In consequence, these outcomes indicate that the magnitude of the SDE phenomenon depends on the ratio of the compound amount to the silica gel amount in the chromatographic purification process. The observed relationship between the SDE and the amount of the separated compound in comparison to the achiral stationary phase may be due to the fact that a smaller quantity has a larger number of unsaturated adsorption centers on a gel, which leads to the intensification of the SDE process. However, when the separation is performed using a larger compound to stationary phase ratio, an increase in gel loading and therefore saturation of the adsorption centers could be observed, which may result in the slowdown of the SDE process. 


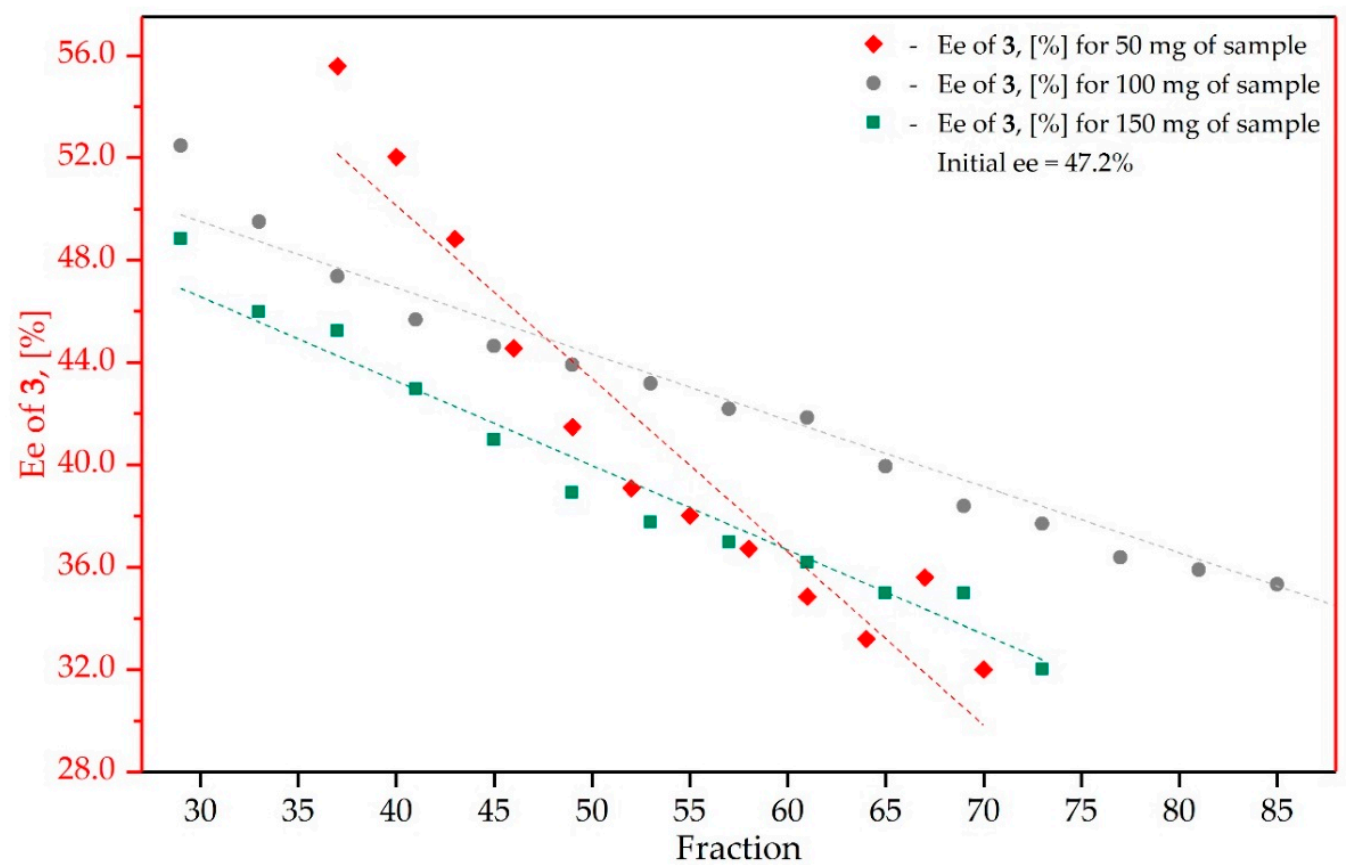

Figure 4. SDE of compound 3 in achiral MPLC conditions: the elution profile. The fitting equation for $50.0 \mathrm{mg}$ of sample is $\mathrm{y}=-0.676 \mathrm{x}+77.190\left(\mathrm{R}^{2}=0.909\right)$ and for $100.0 \mathrm{mg}$ of sample is $\mathrm{y}=-0.352 \mathrm{x}+$ $57.612\left(R^{2}=0.966\right)$ and for $150.0 \mathrm{mg}$ of sample is $y=-0.283 x+58.408\left(R^{2}=0.972\right)$.

\subsection{Study on the SDE of P-Chiral Compounds: tert-Butylphenylphosphinothioic acid (4)}

The influence of the starting material's enantiomeric composition on the magnitude of SDE was examined with the tert-butylphenylphosphinothioic acid (4) (Figure 5). The chosen model compound was earlier synthesized in accordance with the literature procedure [41]. This compound has already found its application as a chiral solvating agent (CSA) for the enantiomeric excess determination by an NMR technique [66]. It is worth noting that the enantiomeric purity of 4 could also be determined by an NMR technique since 4 may simultaneously play a role of the analyzed compound and CSA [67] (Figure S4). The reason for the observed chiral recognition lays in the ability of 4 to act as a donor and an acceptor of hydrogen bonds and hence the formation of homochiral and heterochiral dimeric species (Figure 5). Taking into account the properties of the selected compound, we thought that it would prove an ideal candidate for investigating the dependence of the SDE phenomenon on the initial enantiomeric excess of the tested samples.<smiles>CC(C)(C)P(=O)(S)c1ccccc1</smiles><smiles>CC(C)(C)C1=P2(c3ccccc3)SCSP(=O)(C(C)(C)C)C2(c2ccccc2)SCS1</smiles>

$(S, S)$ - aggregate

4<smiles></smiles>

$(R, S)$ - aggregate

Figure 5. (R)-tert-butylphenylphosphinothioic acid (4): chemical structures on the homochiral and heterochiral aggregates. 
For the purpose of this study, three samples of $(R)-4$ in the amount of $100.0 \mathrm{mg}$ and with the optical purity of $25.0 \%$ ee, $60.0 \%$ ee and $75.0 \%$ ee were prepared, supported on $1.0 \mathrm{~g}$ of silica, and then subjected to the purification process driven by flash chromatography on a $12 \times 150 \mathrm{~mm}$ column filled with $10.0 \mathrm{~g}$ achiral silica gel.

The samples were then eluted in gradient conditions using a mixture of cyclohexane and tert-butyl methyl ether in the ratio from 100/0 to 0/100. The separations were run at the eluent flow rate of $18 \mathrm{~mL} / \mathrm{min}$ and the collected fractions had the volume of $18 \mathrm{~mL}$. To our disappointment, the resulting $\Delta e e$ was not significant and reached only about 3.0\% (Figure 6). The first fractions in which compound 4 was detected by TLC were fractions $7,6,9$ of $25.0,60.0$, and $75.0 \%$ ee, respectively. Compound 4 obtained in the course of the purification exhibited a slightly lower enantiomeric purity in the last fraction (Figures 6 and 7, Table S4).

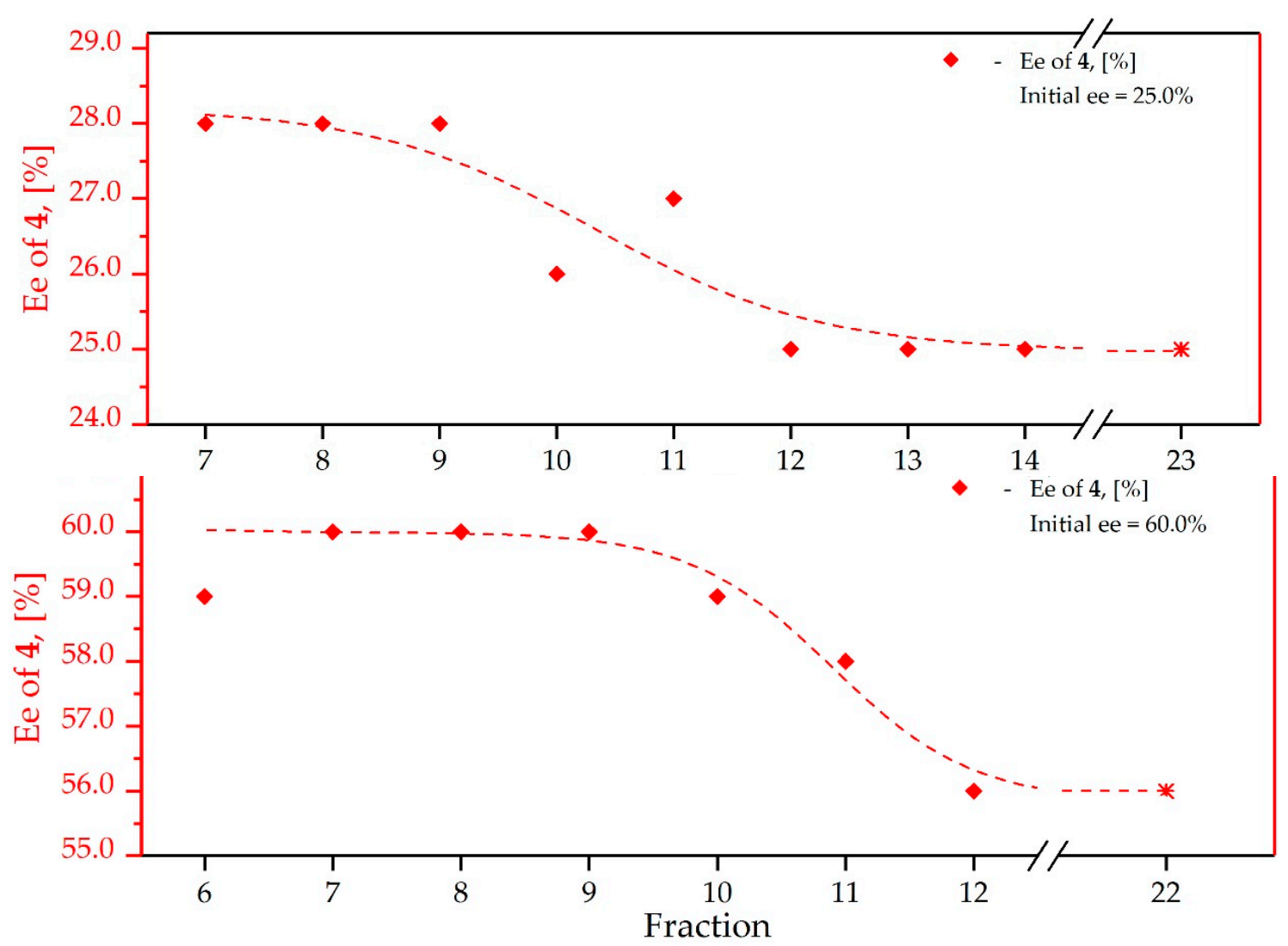

Figure 6. SDE of compound 4 of $25.0 \%$ and $60.0 \%$ ee in achiral MPLC conditions: the elution profiles. The model of the fitting is polynomial.

Since the concentration of the compounds in later fractions was low, those fractions (14-23 for $25.0 \%$ ee sample, $12-22$ for $60.0 \%$ ee sample, and $12-24$ for $75.0 \%$ ee sample) were combined before the ee determination. The distribution of the enantiomer in consequent fractions was determined in the experiment where sample of 4 with $75.0 \%$ ee was used. As it could be seen from Figure 7 and Table S4 the majority of the sample of slightly higher ee was eluted in fractions 9-10, while a less enantioenriched compound was eluted with the next 12 fractions. Such behavior of the compounds may indicate that part of the sample was adsorbed on stationary phase and slowly liberated upon elution. 


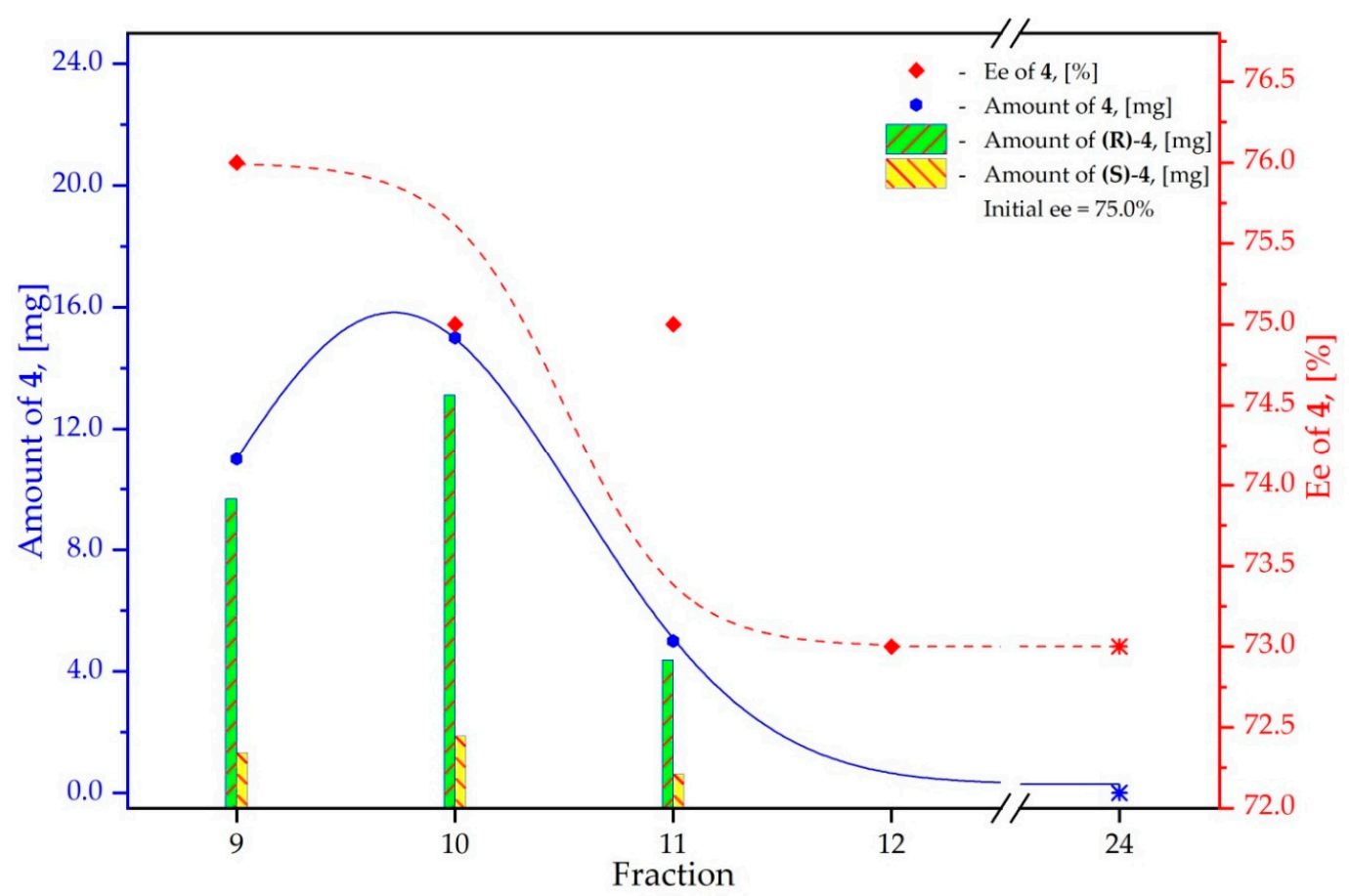

Figure 7. SDE of compound 4 of $75.0 \%$ ee in achiral MPLC conditions: the elution profile. The model of the fitting is polynomial.

An analysis of the collected data showed that in the case of compound 4, even at a low polarity of the eluent, the magnitude of SDE is insignificantly influenced by the enantiomeric purity of the starting material. Additionally, it was observed that the amount of the compound collected after the elution constitutes only around $64.0 \%$ of the weight loaded onto the column. This is caused by strong interactions between the examined compound and the stationary phase and therefore its irreversible adsorption.

In the cases of compounds 1-3, where the separation process was carried out with the isocratic elution, the phenomenon of SDE occurs in a linear manner in time. This may mean that the process takes place at the same speed during the separation procedure. The gradient of a straight line informs us about whether the enrichment or depletion ee of the fraction is observed, and its value represents the speed of these changes. The linear effect of the SDE overtime changes can be explained by the fact that during the isocratic elution, the constants of adsorption and desorption do not change. In the case of gradient elution conditions, as it had been presented for the compounds 4 , the constants of adsorption are change during the elution process and therefore no linear correlation was observed.

\subsection{Study on the SDE of Amino Acid Derivatives: N-Acetyl-Phenylalanine (5)}

Biologically important small molecules are of especial interest in terms of the SDE studies. Therefore, the possibility of enantioseparation of the amino acid derivatives has been studied. $\mathrm{N}$-acetyl-phenylalanine (5) was selected as a model since it is readily available from natural and synthetic amino acid [68], $\mathbf{5}$ is also a product of widely used benchmark asymmetric hydrogenation reaction [69]. In theory, strong intermolecular hydrogen bonding could be responsible for the enantio recognition in the case of amino acids. The simplest possible homochiral (A) and heterochiral (B) dimeric aggregates formed for $\mathrm{N}$-acetyl-phenylalanine are depicted in Figure 8. At the same time, the crystallographic analysis indicates that in the solid state, $\mathrm{N}$-acetyl-amino acids form a more complicated net of hydrogen bonds in which the moieties of amino acids are usually groups in tetrameric cycles (C) [70] [CSD Refcodes: COQHAR, DADGUK01, ACNVAL]. Interestingly, in the presence of protic solvents, the latter could replace one (or more) of the amino acid derivative molecules to form simpler aggregates such as e.g., trimers (D) [CSD Refcode: ACDAHO]. For comparison, similar studies on the 
SDE of $N$-acetylated 1-phenylethylamine [71], and some other amides [72-74], which show a simpler mode of coordination, have already been reported.
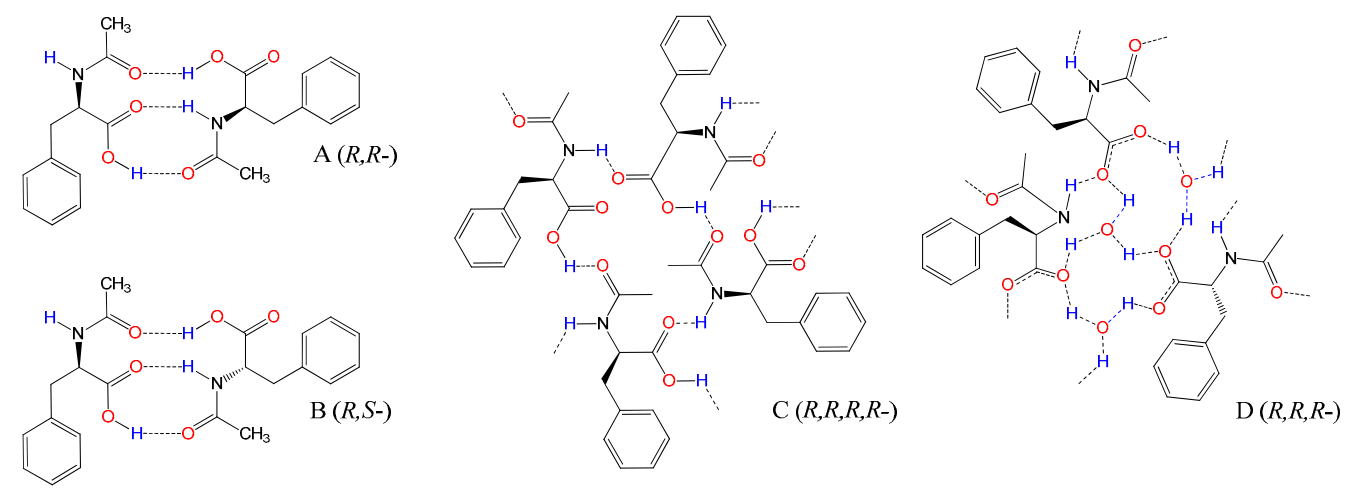

Figure 8. Chemical structures of the possible aggregates of $N$-acetyl-phenylalanine (5).

For the purposes of this study, the samples of $\mathbf{5}$ with the initial value of $e e 50.0 \%$ were evaluated in different eluent systems. First elution was carried out using hexane/i-PrOH $=92 / 8$ and the second series employed dichloroethene as the mobile phase. Since compound $\mathbf{5}$ is not soluble in the mobile phase, $100.0 \mathrm{mg}$ of it was initially dissolved in methanol, and the solvent was evaporated in the presence of about $1.0 \mathrm{~g}$ of silica, then the compounds supported on the stationary phase were loaded at the top of a $12 \times 150 \mathrm{~mm}$ column filled with additional $10.0 \mathrm{~g}$ silica gel. The separations were run at the eluent flow rate of $18 \mathrm{~mL} / \mathrm{min}$ and the collected fractions had the volume of $18 \mathrm{~mL}$. In both cases 26 fractions were collected, compound 5 first appeared in fraction 9 (hexane $\left.{ }^{i-} \mathrm{PrOH}\right)$ and in fraction $12\left(\mathrm{ClC}_{2} \mathrm{H}_{4} \mathrm{Cl}\right)$. The enantiomeric composition of the consecutive fractions was determined be means of a chiral HPLC Chiralpak ${ }^{\circledR}$ OJ-RH column, with the mobile phase using $\mathrm{H}_{2} \mathrm{O} / \mathrm{CH}_{3} \mathrm{CN}=85 / 15$ at the flow rate equal to $0.3 \mathrm{~mL} / \mathrm{min}$ (Figure S5).

The amount of compound 5 and its enantiomeric composition in the consecutive fractions were evaluated and are presented in Table S5. The SDE profiles are presented in Figure 9. Whenever the mixture hexane $/{ }^{i} \mathrm{PrOH}=92 / 8$ was used as the eluent, the value $\Delta e e$ was $5.9 \%$ and the enantiomeric excess of the most enriched fraction 9 exceeded the initial mixture by $3.5 \%$. Using dichloroethane allowed us to reach a greater magnitude of the SDE phenomenon with the $7.5 \%$ value of the $\Delta e e$ parameter. The enantiomeric excess of fraction 12 deviated the initial by $7.2 \%$. The use of an aprotic solvent strongly affected the SDE, and (in contrast to where hexane $/{ }^{i-} \mathrm{PrOH}$ was applied) furnished an elution with the opposite profile, where the initial fractions were enantiomerically depleted and the last ones were enantiomerically enriched.

The total amounts of compound 5 recovered after the purification were $65.7 \%$ (in the case of hexane $/ i-\mathrm{PrOH}$ ), and only $47.0 \%$ (in the case of dichloroethane) of the weight loaded onto the column. The results presented in Table S5 clearly indicate that an irreversible sorption of 5 on the stationary phase was much bigger when the aprotic solvent was used. It also facilitated the formation of heterochiral aggregates $(R, S)$ - in the solution which (being symmetric and less polar-see Figure 10) are eluting faster to form first fractions of a lower $e e$. 


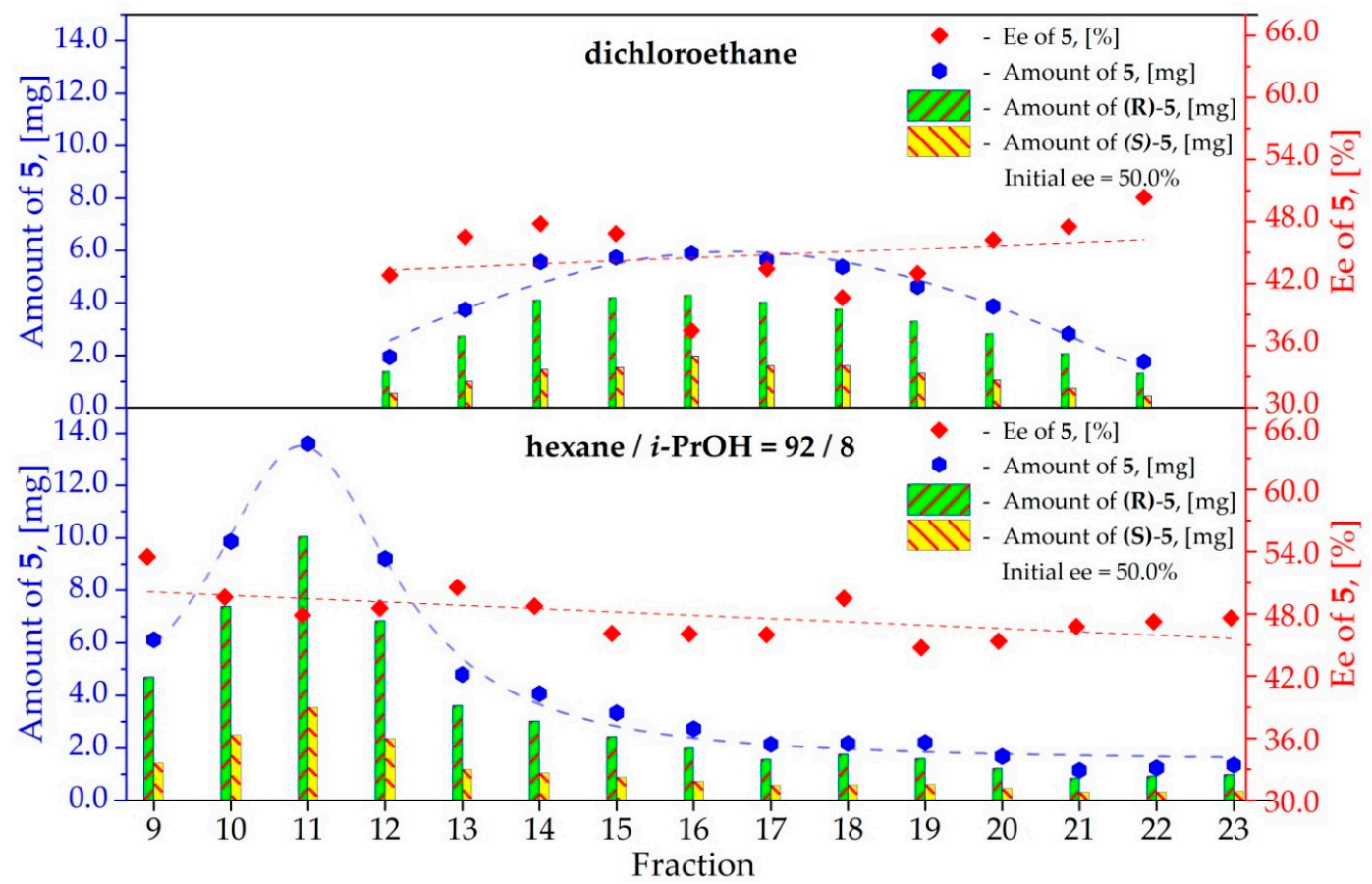

Figure 9. SDE of compound 5 in achiral MPLC conditions: the elution profiles. Fitting equation for dichloroethane is $y=0.730 x+32.462\left(R^{2}=0.261\right)$, and for hexane/i-PrOH is $y=-0.557 x+56.961$ $\left(\mathrm{R}^{2}=0.428\right)$.

The experimental observations correlate well with the thermodynamic analysis of the potential equilibrium between possible stereoisomeric aggregates of 5 (Figure 10). It was found that the bimolecular $(R, S)$-aggregate is more stable than the isomeric $(S, S)$ - or $(R, R)$-aggregates. In particular, an advanced M062x/6-311++G(d,p)(PCM) computational study showed that the differences between the values of Gibbs free energies of the formation of both molecular systems equals 4.0 and $2.5 \mathrm{kcal} / \mathrm{mol}$ respectively (Table 1). Additionally, we analyzed key distances between the substructures within the considered aggregates. It was found that these distances are practically identical in both cases. So, the difference between thermodynamic stabilities is a not a consequence of the power of local intermolecular interactions, but rather a more favorable arrangement of the structural elements in space, which provides more favorable electrostatic interactions and/or lower steric repulsions. Two aggregates are also characterized with different polarities where heterochiral aggregate has a lower dipole moment (Figure 10).

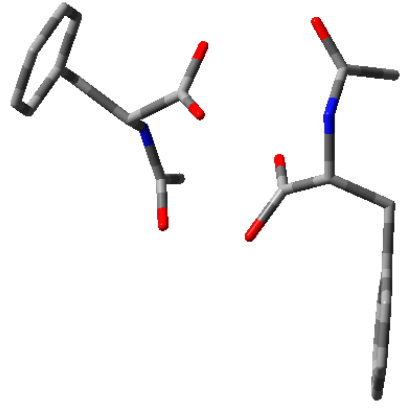

$R, R$-aggregate
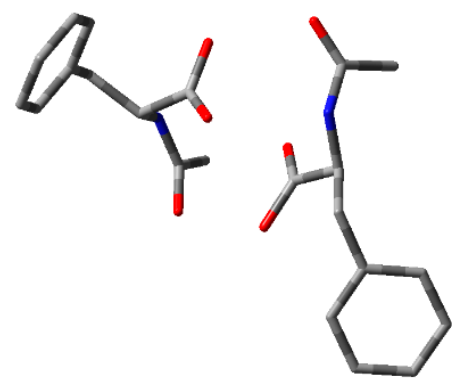

$R, S$-aggregate
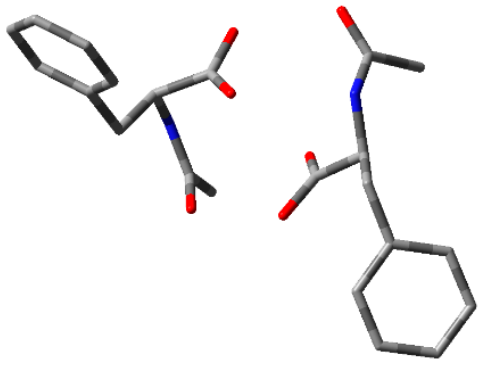

S,S-aggregate

Figure 10. Views of the homo- and heterochiral aggregates of compound 5 in the DCM solution optimized at the M062x/6-311++G(d,p)(PCM) level of theory. 
Table 1. Differences between the heterochiral and homochiral aggregates of compound 5 in the DCM solution optimized at the M062x/6-311++G(d,p)(PCM) level of theory.

\begin{tabular}{cc}
\hline Transition & $\Delta \mathrm{G}[\mathbf{k c a l} / \mathbf{m o l}]$ \\
\hline$(R, S) \rightarrow(S, S)$ & 4.0 \\
\hline$(R, S) \rightarrow(R, R)$ & 2.5 \\
\hline
\end{tabular}

The limited solubility of compound 5 , and its tendency to form diverse and intricate crystalline forms, make the SDE process less efficient and make its study very complicated. Taking that into consideration, a simpler liquid derivative of $\alpha$-amino acids should be selected to eliminate the possible separation via micro crystallization on the stationary phase, and facilitate enantioseparation by limiting the number of possible aggregation models which may be in an equilibrium and have an opposite effect on the SDE efficiency.

\subsection{Study on the SDE of Aamino Acid Derivatives: Methyl Phenylalaninate (6)}

Thus, methyl phenylalaninate (6) was chosen to be examined. The compound was obtained by esterification [75] of $e e=36.0 \%(S)$-phenylalanine in standard conditions for this sequence of the reactions (Scheme 4).

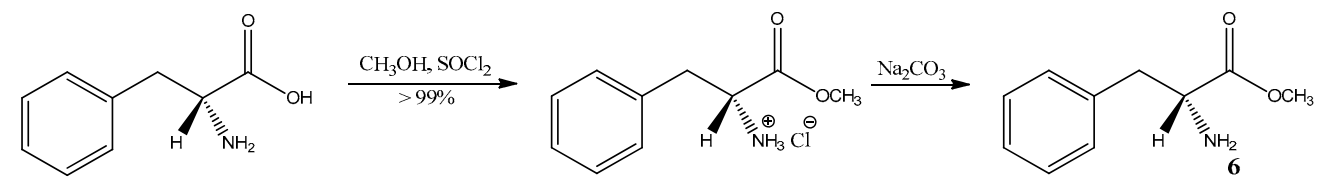

Scheme 4. Synthesis of methyl phenylalaninate (6).

Since 6 is a liquid well soluble in the majority of organic solvents there was no need to support the compound on the stationary phase before the separation. The solution of 6 in $0.5 \mathrm{~mL}$ of the mobile phase with the (S)- enantiomer of $e e=36.0 \%$ in the amount of $100.0 \mathrm{mg}$ was subjected to the separation process on an achiral $12 \times 150 \mathrm{~mm}$ column filled with additional $11.0 \mathrm{~g}$ silica gel and prewashed with the mobile phase. The separations were carried out at the eluent flow rate of $18 \mathrm{~mL} / \mathrm{min}$ and the collected fractions had the volume of $18 \mathrm{~mL}$. In this step, diethyl ether $\left(R_{\mathrm{f}}=0,43\right)$ was used as the mobile phase. The presence of the compound in the obtained fraction was verified on the basis of the TLC technique. At first compound 6 appeared in fraction 12. The optical purity of the fractions was determined based on the ${ }^{1} \mathrm{H}$ and ${ }^{13} \mathrm{C}$ NMR techniques using, as CSA, $100 \mathrm{~mol} \%$ of enantiomericaly pure 4 (Figure S6).

In the case of ${ }^{13} \mathrm{C}$ NMR, two sets of signals corresponding to the $\mathrm{OCH}_{3}(51.41 / 51.45 \mathrm{ppm})$ and $\mathrm{NCH}(54.29 / 54.34 \mathrm{ppm})$ carbon atoms were analyzed, while in the case of ${ }^{1} \mathrm{H}$ NMR only signals corresponding to $\mathrm{NCH}$ proton at $3.95 / 4.01(\mathrm{t}, J=6.5 \mathrm{~Hz}) \mathrm{ppm}$ were used for the ee calculation (Figure S6). The individual optical purities, measured by NMR, and their average values are shown in Table S6.

As a result of this separation (Figure 11, and Table S6), 11 fractions were collected, in which the total amount of the compound was $68.1 \mathrm{mg}$ (where: the mass of the $(R)$-enantiomer was $21.8 \mathrm{mg}$, and the mass of the $S$ enantiomer was $46.3 \mathrm{mg}$ ). This means that during the separation $31.0 \mathrm{mg}$ of compound 6 was permanently adsorbed on the stationary phase, including $10.2 \mathrm{mg}$ of the $(R)$-enantiomer and $21.7 \mathrm{mg}$ of the $(S)$-enantiomer. The $\Delta e e$ value for this separation was $13.8 \%$ ee $(S)$. The differentially calculated enantiomeric composition of compound $\mathbf{6}$ irreversibly adsorbed on the stationary phase is the same as for the initial sample and constitutes $36.0 \%$ ee (S), which means that the adsorption process is not selective and the amount of the adsorbed enantiomer depends only on the initial composition of the starting mixture. 


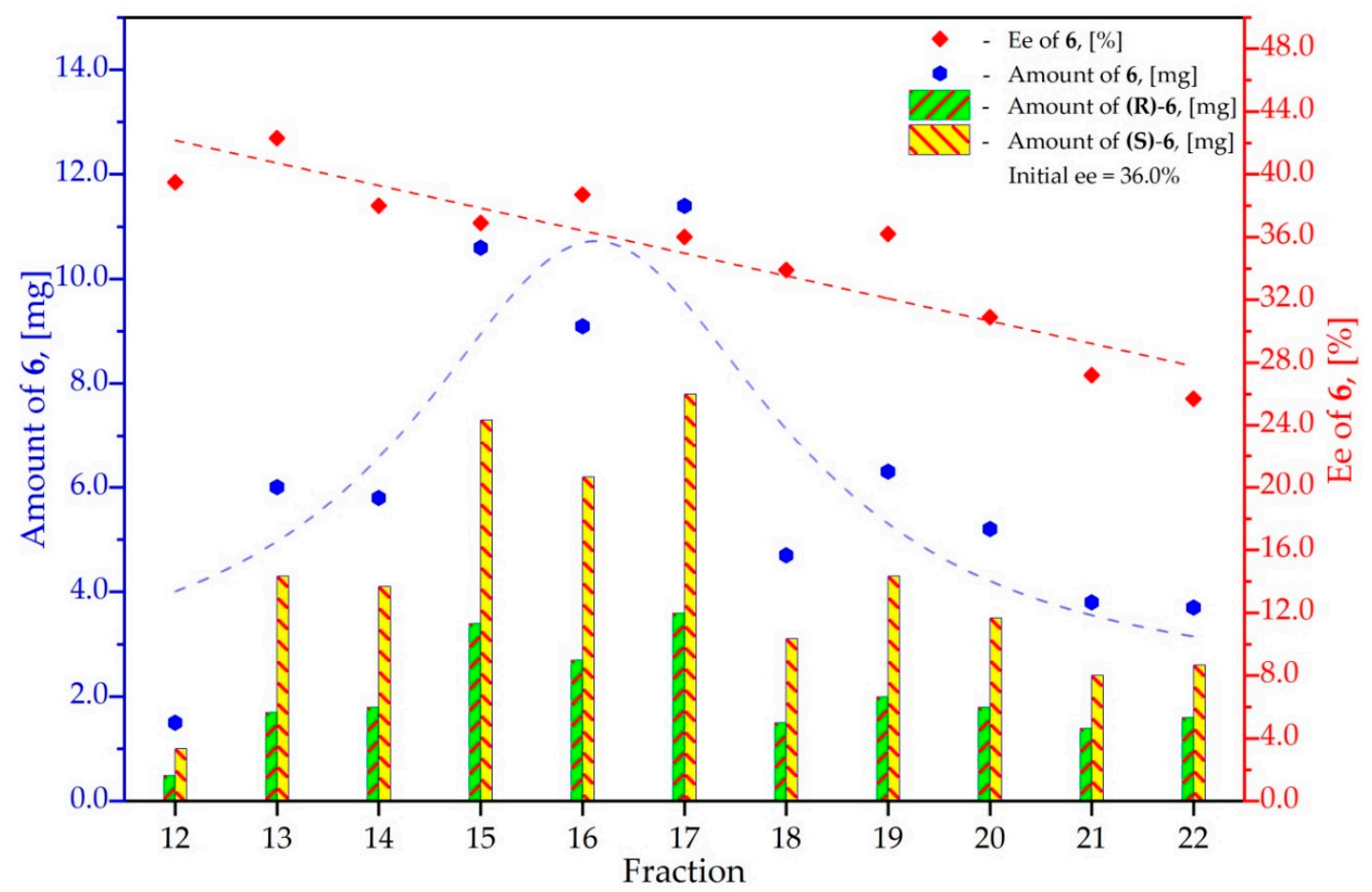

Figure 11. SDE of compound 6 using diethyl ether: the elution profile. The fitting equation is $y=$ $-1.426 x+59.275\left(R^{2}=0.836\right)$.

Since the initial compound is not racemic, its irreversible nonstereoselective adsorption leads to a change in the column's character from achiral to chiral. The enantiomeric purity of the chiral selector on a newly-formed chiral surface depends on the enantiomeric composition of the mixture during loading and on the adsorptive capacity of the stationary phase. Such a newly-created chiral column differentiates the enantiomers of the chromatographed compound and causes their natural separation. This could be a good alternative explanation of the SDE phenomenon under chromatographic condition.

In order to confirm this hypothesis and explain the mechanism of the SDE process, the following experiments were carried out. Compound 6 of the same initial enantiocomposition $(36.0 \%$ ee, $(S)$ was eluted by tert-butyl methyl ether. In the first separation experiment, $100.0 \mathrm{mg}$ of 6 with an initial purity of $36.0 \%$ ee $(S)$ in $0.5 \mathrm{~mL}$ of hexane was injected into a $12 \times 150 \mathrm{~mm}$ column packed with $11.0 \mathrm{~g}$ of spherical achiral silica gel, washed with the mobile phase. The separation was performed using tert-butyl methyl ether (MTBE) as the mobile phase $\left(R_{\mathrm{f}}=0,4\right)$ and the eluent flow rate of $18 \mathrm{~mL} / \mathrm{min}$, $18 \mathrm{~mL}$ of the fractions were collected. As a result of this separation, 8 fractions containing 6 were obtained (fractions from 7 to 14). The presence of the compound in the fractions was verified based on the TLC technique. Then, when no more compound was found in the subsequent fractions collected, the column was used for the second separation experiment, where the same portion of compound $\mathbf{6}$ (as in the case of the first separation) was injected. The second separation experiment was carried out using the same mobile phase MTBE, as in the first separation process. As a result of this separation, 10 fractions were collected (fractions from 36 to 45). The optical purity of the fractions collected in the first and second separation experiments was determined based on the ${ }^{1} \mathrm{H}^{13} \mathrm{C}$ NMR spectroscopic technique with CSA 4. The data collected in the course of both experiments was analyzed, and is presented in Table S7, while the elution profiles are shown in Figure 12. 


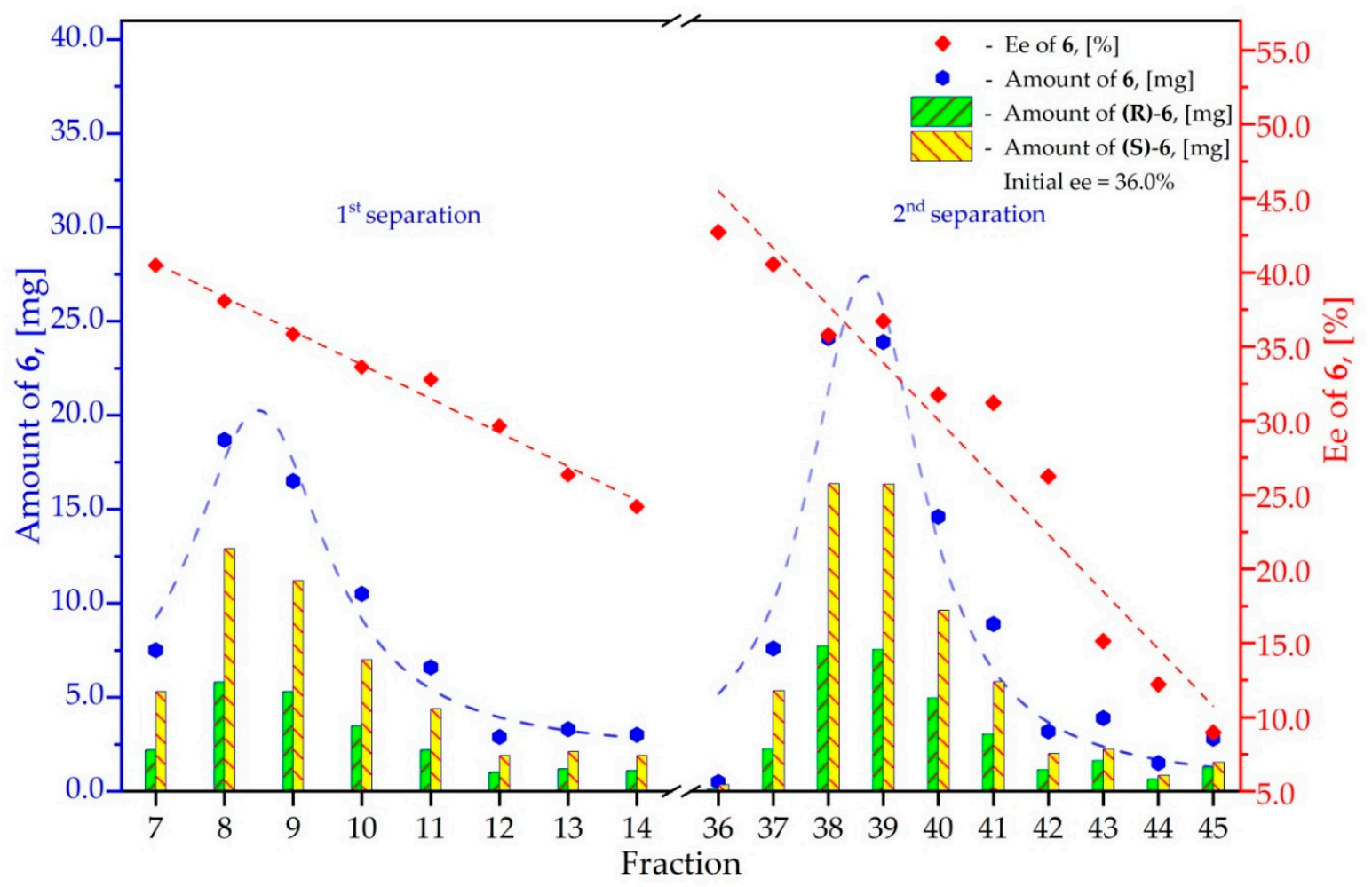

Figure 12. SDE of compound 6 by tert-butyl methyl ether: the elution profile. The fitting equation for 1st separation is $\mathrm{y}=-2.284 \mathrm{x}+56.615\left(\mathrm{R}^{2}=0.988\right)$ and for 2 nd separation is $\mathrm{y}=-3.863 \mathrm{x}+184.578$ $\left(R^{2}=0.936\right)$.

As a result of the first separation experiment, 8 fractions were obtained in which the total mass of the compound was $68.0 \mathrm{mg}(68.0 \%$ of initial), where the calculations were as follows: the amount of the $(R)$-enantiomer was $22.3 \mathrm{mg}$ and the amount of the $(S)$-enantiomer was $46.7 \mathrm{mg}$ (of $35.1 \%$ ee $(S)$, calculated on the basis of each fraction). The value of the $\Delta e e$ for the first separation experiment was $16.0 \%$, and the most enriched fraction 7 ee equaled $40.5 \%$, while the less enriched fraction 14 equaled $24.2 \%$. This means that during the first separation $31 \mathrm{mg}(31.0 \%$ ) of compound 6 (of $37.0 \%$ ee (S)) was permanently adsorbed on the stationary phase, including $9.7 \mathrm{mg}$ of the $(R)$-enantiomer and $21.3 \mathrm{mg}$ of the $(S)$-enantiomer. In the first separation experiment, similarly as it had been observed in the case of the elution with diethyl ether, during the chromatographic process part of the eluted compounds $(31.0 \mathrm{mg}$ ) was adsorbed on the stationary phase not enantio-selectively with the ratio $R / S$ depending only on the initial composition of the mixture.

Notably, in the case of the second separation experiment, the same column was used, but with already adsorbed compound $\mathbf{6}$. The total amount of $\mathbf{6}$ collected after the separation was much higher and equal to $91.0 \mathrm{mg}(91.0 \%)$. The weighted arithmetic mean ratio of the $(R)$-enantiomer to the (S)-enantiomer was 30.7 to $60.3 \mathrm{mg}(33.0 \%$ ee (S)). The value of the $\Delta e e$ for this separation calculated based on the ee of fraction $36(42.7 \%)$ and fraction $45(9.0 \%)$ equaled $33.7 \%$. Thus, only $9.0 \mathrm{mg}(9.0 \%)$ of compound 6 of $71.0 \%$ ee (S) was lost on the stationary phase.

The efficiency of the second separation experiment was therefore notably higher than of the first one. The simple rationalization of this phenomenon could be as follows: the effect can be explained by the formation of a new interaction between the enantiomers and the modified stationary phase which formed after part of the chiral compound was adsorbed on the stationary phase surface. The homochiral and heterochiral aggregates are formed during the separation between the free compound in the mobile phase and the chiral selectors adsorbed on the stationary phase. Such process follows the rule of three points (Pirkle rule) [76,77]. According to this principle, for the chiral separation of enantiomers, it is necessary to have a minimum of three interactions between the enantiomers and 
the chiral selector, as shown in Figure 13, while at least one of these interactions depends on the configuration of the chiral centers of the selector and the selectand. The separation of the enantiomers by HPLC with the chiral stationary phases depends on the formation of transient diastereoisomeric linkages (in our case they are homochiral and heterochiral aggregates). Different stabilities of these complexes result in the possibility of the chiral separation of enantiomers.
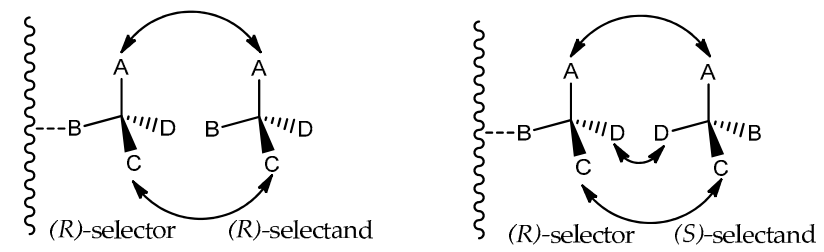

Figure 13. The model of the interactions between selectand and chiral selector.

The efficiency of the enentiomer separation can be explained based on the quantumchemical calculations, as in the case of 6 . In particular, the M062x/6-311++G(d,p) computational study showed that the homochiral aggregate is more stable than the isomeric heterochiral one (Table 2). The model of coordination was based on crystallographic data [70] [CSD Refcodes: IWOMIS] where hydrogen bonds $\mathrm{NH}-\mathrm{O}=\mathrm{C}$ with the bond length of $2.28 \AA$ were responsible for the crystal net formation. Next, the quantumchemical study confirmed that the less polar homochiral aggregates (Figure 14) are eluting faster than the heterochiral ones. The DFT calculation also indicated that the thermodynamic stabilities of the formed aggregates are dependent on the arrangement of their structural elements in space.

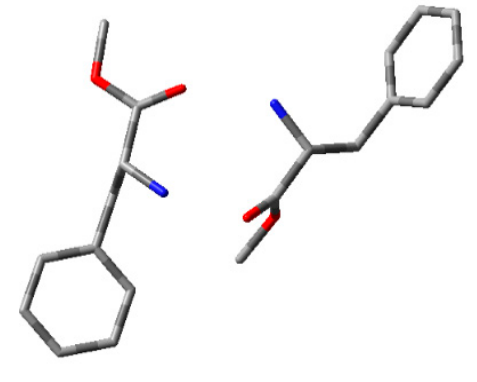

$R, R$-aggregate

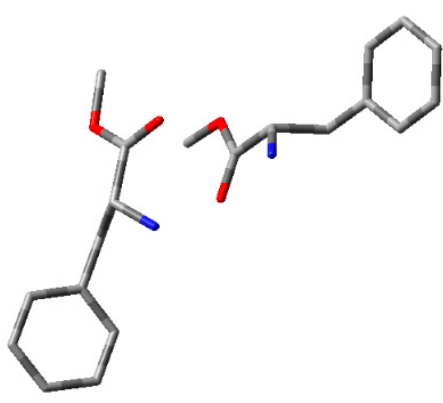

$R, S$-aggregate

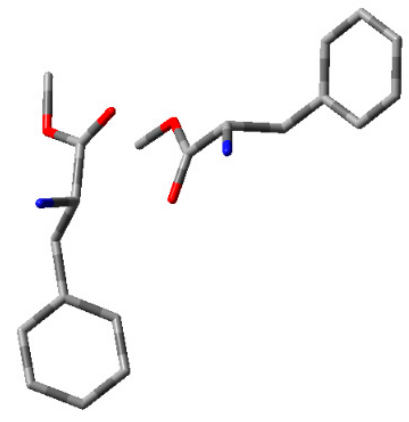

S,S-aggregate

Figure 14. Views of the homo- and heterochiral aggregates of compound 6 in the DCM solution optimized at the M062x/6-311++G(d,p)(PCM) level of theory.

Table 2. Differences between heterochiral and homochiral aggregates of compound 6 in the DCM solution optimized at the M062x/6-311++G(d,p)(PCM) level of theory.

\begin{tabular}{cc}
\hline Transition & $\Delta \mathbf{G}[\mathbf{k c a l} / \mathbf{m o l}]$ \\
\hline$(R, R) \rightarrow(R, S)$ & 2.2 \\
\hline$(S, S) \rightarrow(R, S)$ & 3.3 \\
\hline
\end{tabular}

\section{Conclusions}

The data presented above indicate that the optical purification approach based on SDE has a potential and in some cases could be applied as an alternative to the conventional procedures of chiral separations. The SDE phenomenon of six non-racemic compounds on achiral silica gel was explored, and some correlation between the eluent used, ratio of the compounds to the stationary phases and compound structures was found to influence the magnitude of the enantiomer self-disproportionation process. An explanation of a possible mechanism of this phenomenon, which involves the in situ formation of the chiral surface on the achiral stationary phase, was also provided. The presented 
results may mean that the SDE phenomenon should not only be explained by the formation of the aggregates between the enantiomers in a mobile phase, but also by the change of the column character from achiral to chiral. Since crystallization and sublimation are purification methods applicable only for enantiomerically enriched crystalline samples, SDE-based chromatographic methodology could be applied as a general tool for practical enantiopurification of organic compounds enriched in one of the enantiomers. Such a self enantio-enriching process may take place in nature, and could be responsible for the formation of the first chiral non-racemic compounds on the early Earth.

Additionally, it worth mentioning that, taking into consideration the fact that in many publications on asymmetric synthesis, the optical purity of the furnished products exceeds $99 \% e e$, it is important to measure the enantiomeric purity before any purification of the product obtained in an asymmetric reaction to achieve reliable results of the measurement of the level of an asymmetric induction.

From a practical point of view, to facilitate a SDS effect, is may be recommend to use an aprotic solvents of low polarity and higher ratio of the stationary phase to the separated compound. The mobile phases should have a $R_{f}$ value in the range of 0.2-0.4 and the resulting TLC spot should be wider rather than narrow. Probably, better separations may be obtained in cases of substances forming weak intermolecular hydrogen bonds.

Supplementary Materials: Supplementary materials can be accessed at: http://www.mdpi.com/2073-8994/11/5/680/s1 .

Author Contributions: Conceptualization, O.M.D.; methodology, O.M.D. and M.B.; investigation, O.M.D., M.B., K.K., R.J., W.K., S.K.; writing—original draft preparation, O.M.D., M.B., D.S.; writing-review and editing, O.M.D.; visualization, M.B., O.M.D.; supervision, O.M.D.; inspiration and discussions: K.M.P.

Funding: This research received no external funding. The research was carried out in part using the PLGrid ('Prometheus' cluster) infrastructure (ACK 'Cyfronet' in Cracov), and the equipment purchased thanks to the financial support of the European Regional Development Fund under the framework of the Operational Program Development of Eastern Poland 2007-2013 (Contract No. POPW.01.03.00-06-009/11-00, equipping the laboratories of the Faculties of Biology and Biotechnology, Mathematics, Physics and Informatics, and Chemistry for studies of biologically active substances and environmental samples) as well as the Polish National Science Centre research grant (2012/05/B/ST5/00362).

Conflicts of Interest: The authors declare no conflict of interest.

\section{References}

1. Carreira, E.M.; Yamamoto, H. Comprehensive Chirality, 1st ed.; Elsevier: Amsterdam, The Netherlands, 2012; ISBN 978-008-095-167-6.

2. Hegstrom, R.A.; Kondepudi, D.K. The Handedness of the Universe. Sci. Am. 1990, 262, 108-115. [CrossRef]

3. Lubec, G.; Rosenthal, G.A. Amino Acids, Chemistry, Biology and Medicine; ESCOM: Leiden, The Netherlands, 1990; ISBN 9072199049.

4. Ernst, B.; Hart, G.W.; Sinay, P. Biosynthesis and Degradation of Glycoconjugates; Wiley-VCH: Chichester, UK, 2000; ISBN 352-729-511-9.

5. Gawley, R.E.; Aubé, J. Principles of Asymmetric Synthesis, 2nd ed.; Elsevier: Oxford, UK, 2012; ISBN 978-008-044-860-2.

6. Cohen, J. Getting all turned around over the origins of life on earth. Science 1995, 267, 1265-1266. [CrossRef]

7. McCombs, C. The Extraterrestrial Search for the Origin of Homochirality. Creat. Res. Soc. Q. 2014, 51, 5-13.

8. Rauchfuss, H.; Mitchell, T.N. Chemical Evolution and the Origin of Life by Horst Rauchfuss; Springer: Berlin/Heidelberg, Germany, 2008; ISBN 978-354-078-823-2.

9. McGuire, B.A.; Carroll, P.B.; Loomis, R.A.; Finneran, I.A.; Jewell, P.R.; Remijan, A.J.; Blake, G.A. Discovery of the interstellar chiral molecule propylene oxide (CH3CHCH2O). Science 2016, 352, 1449-1452. [CrossRef]

10. Cooper, G.; Reed, C.; Nguyen, D.; Carter, M.; Wang, Y. Detection and formation scenario of citric acid, pyruvic acid, and other possible metabolism precursors in carbonaceous meteorites. Proc. Natl. Acad. Sci. USA 2011, 108, 14015-14020. [CrossRef] [PubMed]

11. Angel, J.R.P.; Illing, R.; Martin, P.G. Circular polarization of twilight. Nature 1972, 238, 389-390. [CrossRef]

12. Wolstencroft, R.D. Terrestiral and Astronomical Sources of Circular Polarisation: A fresh look at the origin of Homochirality on Earth. Bioastron. Life Stars 2004, 213, 154-158. 
13. Garay, A.S.; Ahigren-Beckendorf, J.A. Differential interaction of chiral $\beta$-particles with enantiomers. Nature 1990, 346, 451-453. [CrossRef]

14. Rikken, G.L.J.A.; Raupach, E. Enantioselective magnetochiral photochemistry. Nature 2000, 405, $932-935$. [CrossRef]

15. Soai, K.; Osanai, S.; Kadowaki, K.; Yonekubo, S.; Shibata, T.; Sato, I. d- and 1-Quartz-Promoted Highly Enantioselective Synthesis of a Chiral Organic Compound. J. Am. Chem. Soc. 1999, 121, 11235-11236. [CrossRef]

16. Tang, L.; Shi, L.; Bonneau, C.; Sun, J.; Yue, H.; Ojuva, A.; Lee, B.L.; Kritikos, M.; Bell, R.G.; Bacsik, Z.; et al. A zeolite family with chiral and achiral structures built from the same building layer. Nat. Mater. 2008, 7, 381-385. [CrossRef] [PubMed]

17. Nógrádi, M.; Fogassy, E.; Pálovics, E.; Schindler, J. Resolution of Enantiomers by Non-Conventional Methods. Synthesis 2005, 10, 1555-1568.

18. Faigl, F.; Fogassy, E.; Nógrádi, M.; Pálovics, E.; Schindler, J. Strategies in optical resolution: A practical guide. Tetrahedron Asymmetry 2008, 19, 519-536. [CrossRef]

19. Nag, A. Asymmetric Synthesis of Drugs and Natural Products; CRC Press: Boca Raton, FL, USA, 2018; ISBN 978-113-803-361-0.

20. Lough, W.J.; Wainer, I.W. Chirality in Natural and Applied Science; CRC Press: Boca Raton, FL, USA, 2002; ISBN 084-932-434-3.

21. Kurihara, N.; Miyamoto, J. Chirality in Agrochemicals; Wiley: New York, NY, USA, 1998; ISBN 047-198-121-4.

22. Kitzerow, H.-S.; Bahr, C. Chirality in Liquid Crystals; Springer: New York, NY, USA, 2001; ISBN 038-798-679-0.

23. Li, Q. Photoactive Functional Soft Materials: Preparation, Properties, and Applications; Wiley: Blackwell, UK, 2019; ISBN 978-352-781-676-7.

24. Mason, S.F. Optical rotatory power. Q. Rev. Chem. Soc. 1963, 17, 20-66. [CrossRef]

25. Schellman, J.A. Circular dichroism and optical rotation. Chem. Rev. 1975, 75, 323-331. [CrossRef]

26. Lloyd, D.K.; Goodall, D.M. Polarimetric detection in high-performance liquid chromatography. Chirality 1989, 1, 251-264. [CrossRef]

27. Roussel, C.; Del Rio, A.; Pierrot-Sanders, J.; Piras, P.; Vanthuyne, N.C. Chiral liquid chromatography contribution to the determination of the absolute configuration of enantiomers. J. Chromatogr. A 2004, 1037, 311-328. [CrossRef]

28. Pakulski, Z.; Demchuk, O.M.; Kwiatosz, R.; Osiński, P.W.; Świerczyńska, W.; Pietrusiewicz, K.M. The classical Kagan's amides are still practical NMR chiral shift reagents: Determination of enantiomeric purity of P-chirogenic phospholene oxides. Tetrahedron Asymmetry 2003, 14, 1459-1462. [CrossRef]

29. Demchuk, O.M.; Świerczynska, W.; Pietrusiewicz, K.M.; Woźnica, M.; Wójcik, D.; Frelek, J. A convenient application of the NMR and CD methodologies for the determination of enantiomeric ratio and absolute configuration of chiral atropoisomeric phosphine oxides. Tetrahedron Asymmetry 2008, 19, 2339-2345. [CrossRef]

30. Goering, H.L.; Eikenberry, J.N.; Koermer, G.S. Tris[3-(trifluoromethylhydroxymethylene)-dcamphorato]europium(III) a chiral shift reagent for direct determination of enantiomeric compositions. J. Am. Chem. Soc. 1971, 3, 5913-5914. [CrossRef]

31. McCreary, M.D.; Lewis, D.W.; Wernick, D.L.; Whitesides, G.M. Determination of enantiomeric purity using chiral lanthanide shift reagents. J. Am. Chem. Soc. 1974, 96, 1038-1054. [CrossRef]

32. Kolodiazhnyi, O.I.; Demchuk, O.M.; Gerschkovich, A.A. Application of the dimenthyl chlorophosphite for the chiral analysis of amines, amino acids and peptides. Tetrahedron Asymmetry 1999, 10, 1729-1732. [CrossRef]

33. Seco, J.M.; Latypov, S.; Quiñoá, E.; Riguera, R. New chirality recognizing reagents for the determination of absolute stereochemistry and enantiomeric purity by NMR. Tetrahedron Lett. 1994, 18, 2921-2924. [CrossRef]

34. Wenzel, T.J.; Wilcox, J.D. Chiral reagents for the determination of enantiomeric excess and absolute configuration using NMR spectroscopy. Chirality 2003, 15, 256-270. [CrossRef] [PubMed]

35. Soloshonok, V.A. Remarkable Amplification of the self-disproportionation of enantiomers on achiral-phase chromatography columns. Angew. Chem. Int. Ed. 2006, 45, 766-769. [CrossRef]

36. Soloshonok, V.A.; Berbasov, D.O. Self-disproportionation of enantiomers of (R)-ethyl 3-(3,5dinitrobenzamido)-4,4,4-trifluorobutanoate on achiral silica gel stationary phase. J. Fluorine Chem. 2006, 127, 597-603. [CrossRef] 
37. Cundy, K.C.; Crooks, P.A. Unexpected phenomenon in the high-performance liquid chromatographic analysis of racemic 14C-labelled nicotine: Separation of enantiomers in a totally achiral system. J. Chromatogr. A 1983, 281, 17-33. [CrossRef]

38. Soloshonok, V.; Sorochinsky, A.; Aceña, J. Self-Disproportionation of Enantiomers of Chiral, Non-Racemic Fluoroorganic Compounds: Role of Fluorine as Enabling Element. Synthesis 2012, 45, 141-152. [CrossRef]

39. Faigl, F.; Fogassy, E.; Nógradi, M.; Palovics, E.; Schindler, J. Separation of non-racemic mixtures of enantiomers: An essential part of optical resolution. Org. Biomol. Chem. 2010, 8, 947-959. [CrossRef]

40. Garin, D.L.; Greco, D.J.C.; Kelley, L. Enhancement of optical activity by fractional sublimation. An alternative to fractional crystallization and a warning. J. Org. Chem. 1977, 42, 1249-1251. [CrossRef]

41. Omelańczuk, J.; Mikołajczyk, M. Chiral t-butylphenylphosphinothioic acid: A useful chiral solvating agent for direct determination of enantiomeric purity of alcohols, thiols, amines, diols, aminoalcohols and related compounds. Tetrahedron Asymmetry 1996, 7, 2687-2694. [CrossRef]

42. Tsai, T.L.; Herman, K.; Hug, E.; Rohde, B.; Dreiding, A.S. Enantiomer-Differentiation Induced by an Enantiomeric Excess during Chromatography with Achiral Phases. Helv. Chem. Acta 1985, 68, 2238-2243. [CrossRef]

43. Viedma, C.; Noorduin, W.L.; Ortiz, J.E.; de Torres, T.; Cintas, P. Asymmetric amplification in amino acid sublimation involving racemic compound to conglomerate conversion. Chem. Commun. 2011, 47, 671-673. [CrossRef] [PubMed]

44. Charles, R.; Gil-Av, E. Self-amplification of optical activity by chromatography on an achiral adsorbent. J. Chromatogr. A 1984, 298, 516-520. [CrossRef]

45. Dobashi, A.; Motoyama, Y.; Kinoshita, K.; Hara, S.; Fukasaku, N. Self-induced chiral recognition in the association of enantiomeric mixtures on silica gel chromatography. Anal. Chem. 1987, 59, 2209-2211. [CrossRef]

46. Frost, C.G.; Howarth, J.; Williams, J.M.J. Selectivity in palladium catalyzed allylic substitution. Tetrahedron Asymmetry 1992, 3, 1089-1122. [CrossRef]

47. Dawson, G.J.; Frost, C.G.; Williams, J.M.J. Asymmetric palladium catalysed allylic substitution using phosphorus containing oxazoline ligands. Tetrahedron Lett. 1993, 34, 3149-3150. [CrossRef]

48. Demchuk, O.M.; Kielar, K.; Pietrusiewicz, K.M. Rational design of novel ligands for environmentally benign cross-coupling reactions. Pure Appl. Chem. 2011, 3, 633-644. [CrossRef]

49. Demchuk, O.M.; Kapłon, K.; Kącka, A.; Pietrusiewicz, K.M. The utilization of chiral phosphorus ligands in atroposelective cross-coupling reactions. Phosphorus Sulfur Silicon Relat. Elem. 2016, 191, 180-200. [CrossRef]

50. Jasiński, R.; Demchuk, O.M.; Babyuk, D. A Quantum-Chemical DFT Approach to Elucidation of the Chirality Transfer Mechanism of the Enantioselective Suzuki-Miyaura Cross-Coupling Reaction. J. Chem. 2017, 2017, 3617527. [CrossRef]

51. Zhang, D.; Wang, Q. Palladium catalyzed asymmetric Suzuki-Miyaura coupling reactions to axially chiral biaryl compounds: Chiral ligands and recent advances. Coord. Chem. Rev. 2015, 286, 1-16. [CrossRef]

52. Sawai, K.; Tatumi, R.; Nakahodo, T.; Fijihara, H. Asymmetric Suzuki-Miyaura coupling reactions catalyzed by chiral palladium nanoparticles at room temperature. Angew. Chem. 2008, 120, 7023-7025. [CrossRef]

53. Braun, M.; Devant, R. (R)- and (S)-2-acetoxy-1,1,2-triphenylethanol-Effective synthetic equivalents of a chiral acetate enolate. Tetrahedron Lett. 1984, 25, 5031-5034. [CrossRef]

54. Tempkin, O.; Abel, S.; Chen, C.P.; Underwood, R.; Prasad, K.; Chen, K.M.; Repic, O.; Blacklock, T.J. Asymmetric synthesis of 3,5-dihydroxy-6(E)-heptenoate-containing HMG-CoA reductase inhibitors. Tetrahedron 1997, 53, 10659-10670. [CrossRef]

55. Zhao, Y.; Truhlar, D.G. Density Functionals with Broad Applicability in Chemistry. Acc. Chem. Res. 2008, 41, 157-167. [CrossRef]

56. Zhao, Y.; Truhlar, D.G. The M06 suite of density functionals for main group thermochemistry, thermochemical kinetics, noncovalent interactions, excited states, and transition elements: Two new functionals and systematic testing of four M06-class functionals and 12 other functionals. Theor. Chem. Acc. 2007, 120, 215-241.

57. Frisch, M.J.; Trucks, G.W.; Schlegel, H.B.; Scuseria, G.E.; Robb, M.A.; Cheeseman, J.R.; Scalmani, G.; Barone, V.; Mennucci, B.; Petersson, G.A. Gaussian 09, Revision B.01; Gaussian Inc.: Wallingford, UK, 2010.

58. Demchuk, O.M.; Jasiński, R.; Pietrusiewicz, K.M. New Insights into the Mechanism of Reduction of Tertiary Phosphine Oxides by Means of Phenylsilane. Heteroatom Chem. 2015, 26, 441-448. [CrossRef] 
59. Pietrusiewicz, K.; Szwaczko, K.; Mirosław, B.; Dybała, I.; Jasiński, R.; Demchuk, O.M. New Rigid Polycyclic Bis(phosphane) for Asymmetric Catalysis. Molecules 2019, 24, 571. [CrossRef]

60. Mirosław, B.; Babyuk, D.; Łapczuk-Krygier, A.; Kącka-Zych, A.; Demchuk, O.M.; Jasiński, R. Regiospecific formation of the nitromethyl-substituted 3-phenyl-4,5-dihydroisoxazole via [3 + 2]cycloaddition. Monatshefte für Chemie Chem. Mon. 2018, 149, 1877-1884. [CrossRef]

61. Jin, M.-J.; Takale, V.B.; Sarkar, M.S.; Kim, Y.-M. Highly enantioselective Pd-catalyzed allylic alkylation using new chiral ferrocenylphosphinoimidazolidine ligands. Chem. Commun. 2006, 6, 663-664. [CrossRef]

62. Martinez, C.R.; Iverson, B.L. Rethinking the term "pi-stacking". Chem. Sci. 2012, 3, 2191-2201. [CrossRef]

63. Demchuk, O.M.; Justyniak, I.; Miroslaw, B.; Jasinski, R. 2-Methoxynaphthylnaphthoquinone and its solvate: Synthesis and structure-properties relationship. J. Phys. Organ. Chem. 2014, 27, 66-73. [CrossRef]

64. Nakashima, E.; Yamamoto, H. Asymmetric Aldol Synthesis: Choice of Organocatalyst and Conditions. Chem. Asian J. 2017, 12, 41-44. [CrossRef] [PubMed]

65. Sakthivel, K.; Notz, W.; Bui, T.; Barbas, C.F. Amino Acid Catalyzed Direct Asymmetric Aldol Reactions: A Bioorganic Approach to Catalytic Asymmetric Carbon-Carbon Bond-Forming Reactions. J. Am. Chem. Soc. 2001, 123, 5260-5267. [CrossRef]

66. Chrzanowski, J.; Krasowska, D.; Urbaniak, M.; Sieroń, L.; Pokora-Sobczak, P.; Demchuk, O.M.; Drabowicz, J. Synthesis of Enantioenriched Aryl-tert-Butylphenylphosphine Oxides via Cross-Coupling Reactions of tert-Butylphenylphosphine Oxide with Aryl Halides. Eur. J. Organ. Chem. 2018, 2018, 4614-4627. [CrossRef]

67. Harger, M.J.P. Chemical shift non-equivalence of enantiomers in the proton magnetic resonance spectra of partly resolved phosphinothioic acids. J. Chem. Soc. Perkin II 1978, 4, 326-331. [CrossRef]

68. Dressen, M.H.C.L.; van de Kruijs, B.H.P.; Meuldijk, J.; Vekemans, J.A.J.M.; Hulshof, L.A. From Batch to Flow Processing: Racemization of N-Acetylamino Acids under Microwave Heating. Organ. Process Res. Dev. 2009, 13, 888-895. [CrossRef]

69. Demchuk, O.M.; Arlt, D.; Jasiński, R.; Pietrusiewicz, K.M. Relationship between structure and efficiency of atropisomeric phosphine ligands in homogeneous catalytic asymmetric hydrogenation. J. Phys. Organ. Chem. 2012, 25, 1006-1011. [CrossRef]

70. Groom, C.R.; Bruno, I.J.; Lightfoot, M.P.; Ward, S.C. The Cambridge Structural Database. Acta Crystallogr. Sect. B Struct. Sci. Cryst. Eng. Mater. 2016, 72, 171-179. [CrossRef]

71. Wzorek, A.; Sato, A.; Drabowicz, J.; Soloshonok, V.A. Self-disproportionation of enantiomers via achiral gravity-driven column chromatography: A case study of $\mathrm{N}$-acyl- $\alpha$-phenylethylamines. J. Chromatogr. A 2016, 1467, 270-278. [CrossRef] [PubMed]

72. Wzorek, A.; Kamizela, A.; Sato, A.; Soloshonok, V.A. Self-Disproportionation of Enantiomers (SDE) via achiral gravity-driven column chromatography of N-fluoroacyl-1-phenylethylamines. J. Fluorine Chem. 2017, 196, 37-43. [CrossRef]

73. Suzuki, Y.; Han, J.; Kitagawa, O.; AceÇa, J.L.; Klika, K.D.; Soloshonok, V.A. Comprehensive examination of the self-disproportionation of enantiomers (SDE) of chiral amides via achiral, laboratory-routine, gravity-driven column chromatography. RCS Adv. 2015, 5, 2988-2993. [CrossRef]

74. Wzorek, A.; Sato, A.; Drabowicz, J.; Soloshonok, V.A.; Klika, K.D. Enantiomeric enrichments via the self-disproportionation of enantiomers (SDE) by achiral, gravity-driven column chromatography: A case study using $N$-(1-Phenylethyl)acetamide for optimizing the enantiomerically pure yield and magnitude of the SDE. Helv. Chem. Acta 2015, 98, 1147-1159. [CrossRef]

75. Mori, M.; Deodato, D.; Kasula, M.; Ferraris, D.M.; Sanna, A.; De Logu, A.; Rizzi, M.; Botta, M. Design, synthesis, SAR and biological investigation of 3-(carboxymethyl)rhodanine and aminothiazole inhibitors of Mycobacterium tuberculosis Zmp1. Bioorg. Med. Chem. Lett. 2018, 28, 637-641. [CrossRef]

76. Pirkle, W.H.; Pochapsky, T.C. Considerations of chiral recognition relevant to the liquid chromatography separation of enantiomers. Chem. Rev. 1989, 89, 347-362. [CrossRef]

77. Polavarapu, P.L. Chiral Analysis-Advances in Spectroscopy, Chromatography and Emerging Methods, 2nd ed.; Elsevier Science: Atlanta, GA, USA, 2018; ISBN 978-044-464-028-4.

(C) 2019 by the authors. Licensee MDPI, Basel, Switzerland. This article is an open access article distributed under the terms and conditions of the Creative Commons Attribution (CC BY) license (http://creativecommons.org/licenses/by/4.0/). 